\title{
ATOMS: ALMA Three-millimeter Observations of Massive Star-forming regions - VII. A catalogue of SiO clumps from ACA observations
}

\author{
Rong Liu, ${ }^{1,2 \star}$ Tie Liu, $\dagger^{3,4}$ Gang Chen, $\ddagger^{5}$ Hong-Li Liu, ${ }^{6}$ Ke Wang, ${ }^{7}$ Jin-Zeng Li, ${ }^{1}$ Xun-Chuan Liu, ${ }^{3,10}$
} Chang Won Lee, ${ }^{8,9}$ Paul F. Goldsmith, ${ }^{20}$ Mika Juvela, ${ }^{11}$ Guido Garay, ${ }^{12}$ Leonardo Bronfman, ${ }^{12}$ Tapas Baug, ${ }^{22}$ Jinhua He, ${ }^{15,21,12}$ Si-Ju Zhang, ${ }^{7}$ Yong Zhang, ${ }^{17}$ Feng-Wei Xu, ${ }^{10}$ Archana Soam, ${ }^{14}$ Zhi-Qiang Shen, ${ }^{3}$ Shanghuo Li, ${ }^{8}$ Lokesh Dewangan, ${ }^{13}$ Chakali Eswaraiah, ${ }^{16}$ Yue-Fang Wu, ${ }^{10}$ Sheng-Li Qin, ${ }^{6}$ L. Viktor Tóth, ${ }^{18}$ Zhiyuan Ren, ${ }^{1}$ Guoyin Zhang ${ }^{1}$ Anandmayee Tej, ${ }^{19}$ Qiuyi Luo, ${ }^{3}$ Jianwen Zhou ${ }^{1,2}$ Chang Zhang ${ }^{1,2}$

${ }^{1}$ National Astronomical Observatories of China, Chinese Academy of Sciences, Beijing, 100012, China

${ }^{2}$ University of Chinese Academy of Sciences, Beijing 100049, Peoples Republic of China

${ }^{3}$ Shanghai Astronomical Observatory, Chinese Academy of Sciences, 80 Nandan Road, Shanghai 200030, Peoples Republic of China

${ }^{4}$ Key Laboratory for Research in Galaxies and Cosmology, Chinese Academy of Sciences, 80 Nandan Road, Shanghai 200030, Peoples Republic of China

${ }^{5}$ China University of Geosciences, Wuhan 430074, China

${ }^{6}$ Department of Astronomy, Yunnan University, Kunming, 650091, PR China

${ }^{7}$ Kavli Institute for Astronomy and Astrophysics, Peking University, 5 Yiheyuan Road, Haidian District, Beijing 100871, China

${ }^{8}$ Korea Astronomy and Space Science Institute, 776 Daedeokdaero, Yuseong-gu, Daejeon 34055, Republic of Korea

${ }^{9}$ University of Science and Technology, Korea (UST), 217 Gajeong-ro, Yuseong-gu, Daejeon 34113, Republic of Korea

${ }^{10}$ Department of Astronomy, Peking University, 100871, Beijing, People's Republic of China

${ }^{11}$ Department of Physics, P.O. Box 64, FI-00014, University of Helsinki, Finland

${ }^{12}$ Departamento de Astronomía, Universidad de Chile, Las Condes, Santiago, Chile

${ }^{13}$ Physical Research Laboratory, Navrangpura, Ahmedabad-380 009, India

${ }^{14}$ SOFIA Science Centre, USRA, NASA Ames Research Centre, MS-12, N232, Moffett Field, CA 94035, USA

${ }^{15}$ Yunnan Observatories, Chinese Academy of Sciences, 396 Yangfangwang, Guandu District, Kunming, 650216, China

${ }^{16}$ Indian Institute of Science Education and Research (IISER) Tirupati, Rami Reddy Nagar, Karakambadi Road, Mangalam (P.O.), Tirupati 517 507, India

${ }^{17}$ School of Physics and Astronomy, Sun Yat-Sen University Zhuhai Campus, Tangjia, Zhuhai 519082, China

${ }^{18}$ Eötvös Loránd University, Department of Astronomy, Pázmány Péter sétány 1/A, H-1117, Budapest, Hungary

${ }^{19}$ Indian Institute of Space Science and Technology, Thiruvanan- thapuram 695 547, Kerala, India

${ }^{20}$ Jet Propulsion Laboratory, California Institute ofTechnology, 4800 Oak Grove Drive, Pasadena, CA 91109, USA

${ }^{21}$ Chinese Academy of Sciences South America Center for Astronomy, National Astronomical Observatories, CAS, Beijing 100101, China

${ }^{22}$ S. N. Bose National Centre for Basic Sciences, Block-JD, Sector-III, Salt Lake City, Kolkata 700106, India

Accepted XXX. Received YYY; in original form ZZZ

\section{ABSTRACT}

To understand the nature of $\mathrm{SiO}$ emission, we conducted ACA observations of the $\mathrm{SiO}$ (2-1) lines toward 146 massive star-forming regions, as part of the ALMA Three-millimeter Observations of Massive Star-forming regions (ATOMS) survey. We detected $\mathrm{SiO}$ emission in $128(87.7 \%)$ sources and identified $171 \mathrm{SiO}$ clumps, 105 of which are spatially separated from $3 \mathrm{~mm}$ continuum emission. A large amount of the $\mathrm{SiO}$ line profiles $(60 \%)$ are non-Gaussian. The velocity dispersion of the $\mathrm{SiO}$ lines ranges from 0.3 to $5.43 \mathrm{~km} \mathrm{~s}^{-1}$. In 63 sources the $\mathrm{SiO}$ clumps are associated with $\mathrm{HII}$ regions characterized by $\mathrm{H} 40 \alpha$ emission. We find that $68 \%$ (116) of the $\mathrm{SiO}$ clumps are associated with strong outflows. The median velocity dispersion of the $\mathrm{SiO}$ line for outflow sources and non-outflow sources is $1.91 \mathrm{~km} \mathrm{~s}^{-1}$ and $0.99 \mathrm{~km} \mathrm{~s}^{-1}$, respectively. These results indicate that outflow activities could be connected to strongly shocked gas. The velocity dispersion and $[\mathrm{SiO}] /\left[\mathrm{H}^{13} \mathrm{CO}^{+}\right]$intensity ratio do not show any correlation with the dust temperature and particle number density of clumps. We find a positive correlation between the $\mathrm{SiO}$ line luminosity and the bolometric luminosity, implying stronger shock activities are associated with more luminous proto-clusters. The $\mathrm{SiO}$ clumps in associations with $\mathrm{H}_{\mathrm{II}}$ regions were found to show a steeper feature in $L_{\mathrm{sio}} / L_{\mathrm{bol}}$. The $\mathrm{SiO}$ line luminosity and the fraction of shocked gas have no apparent evidence of correlation with the evolutionary stages traced by luminosity to mass ratio $\left(L_{\mathrm{bol}} / M\right)$.

Key words: stars: formation - ISM: clouds - ISM: molecules 


\section{INTRODUCTION}

As the main contributors of heavy elements and UV-photon radiation, massive stars play an important role in the evolution of interstellar medium (ISM). In the past decades, studies of massive star formation through multi-wavelengths and high-resolution observations have made great achievements, but there are still difficulties in understanding the formation process because of large distances, short timescales, high extinction, and clustered environments (Zinnecker \& Yorke 2007; Motte et al. 2018). The process of massive star formation itself can also provide strong feedback to the parent clouds and ISM by energetic jets and induced outflows. The jets and outflows interacting with the surrounding medium are ubiquitous at different evolutionary stages in high-mass star formation, such as infrared quiet, infrared bright, and Ultra-Compact (UC) HII stage (Churchwell 2002; Bally 2016; Li et al. 2019a). Thus studies of such shock activities can help us deepen understanding of the massive star formation processes.

A deep understanding of the properties of jets and outflows is crucial in answering the problems in massive star formation (Motte et al. 2018). Molecular lines are powerful tools to study the physical and chemical conditions and the feedback within massive star-forming regions (SFRs). Various molecular emissions trace different layers in the internal structure of massive clumps embedding high-mass stars (Csengeri et al. 2016). Rotational transitions of different molecules provide us temperatures, densities, UV fields, chemical abundances, and gas kinematics of the massive clumps. Molecules such as $\mathrm{CO}$, $\mathrm{SiO}, \mathrm{SO}, \mathrm{HCO}^{+}$, and $\mathrm{CS}$ are good tracers of jets and outflows (Bally 2016). In particular, due to little contamination from the quiescent gas, $\mathrm{SiO}$ emissions have been widely used to study shock activities induced by outflows.

Shocks are a universal phenomenon in SFRs. High velocity shocks $\left(v_{\mathrm{s}} \geq 20 \mathrm{~km} \mathrm{~s}^{-1}\right)$ are caused by powerful outflows and jets from massive young proto-stars (Qiu et al. 2007; Duarte-Cabral et al. 2014; Li et al. 2019b, 2020), while low velocity shocks $\left(v_{\mathrm{S}}<10 \mathrm{~km} \mathrm{~s}^{-1}\right.$ ) could be associated with less powerful outflows (Lefloch et al. 1998), cloud-cloud collisions (Louvet et al. 2016) or large-scale gas inflows (Jiménez-Serra et al. 2010). SiO is an excellent tracer of shocks. In the shocked regions, grain material is destructed via sputtering or vaporization, leading the $\mathrm{Si}$ atoms and $\mathrm{Si}$-bearing molecules to be injected into the gas phase and subsequently oxidized to $\mathrm{SiO}$ (Schilke et al. 1997; Gusdorf et al. 2008b). In active star-forming regions, $\mathrm{SiO}$ abundance is enhanced by up to six orders of magnitudes compared to quiescent regions (Martin-Pintado et al. 1992; Codella et al. 1999). Observations of the $\mathrm{SiO}$ emission lines toward different sources exhibit a variety of profiles. Previous studies reported that the spectra of $\mathrm{SiO}$ usually shows two Gaussian components both corresponding to similar central velocities but different line widths, namely, the broad component and the narrow one (Martin-Pintado et al. 1992; Lefloch et al. 1998). These two SiO components trace different emission regions and may have different origins (Schilke et al. 1997). The broad components are thought to be related to high-velocity shocks from protostellar outflows, and the narrow components are attributed to low-velocity shocks linked to low-velocity motions (see above).

Based on studies targeting the variation of $\mathrm{SiO}$ emission toward different evolutionary stages of star formation, several studies found the brighter $\mathrm{SiO}$ emission are associated with higher luminosity sources (Codella et al. 1999; Liu et al. 2020c), which would be consistent

† E-mail: ddwhcg@cug.edu.cn with the trend that the $\mathrm{SiO}$ abundance in warmer sources is higher than the one in infrared-quiet sources (Miettinen 2014; Gerner et al. 2014). Motte et al. (2007) and Sakai et al. (2010) found that younger sources show brighter $\mathrm{SiO}$ emission than mid-infrared bright sources. The studies of Miettinen et al. (2006), López-Sepulcre et al. (2011), and Sánchez-Monge et al. (2013) are consistent with a decrease in $\mathrm{SiO}$ abundance in more evolved sources. The results that the $\mathrm{SiO}$ abundance have no trend among different evolutionary stages also have been reported by Sanhueza et al. (2012), Leurini et al. (2014), Csengeri et al. (2016), and Li et al. (2019a). Recently, Liu et al. (2021c) reported an increasing trend of the $\mathrm{SiO}$ line luminosity with bolometric luminosity, but they do not see the relation between the $\mathrm{SiO}$ line luminosity and the bolometric luminosity-to-mass ratio. So far, there is no consensus on $\mathrm{SiO}$ abundance variation in different evolutionary stages.

During the past decades, a large of $\mathrm{SiO}$ studies toward massive star-forming regions have been conducted. The $\mathrm{SiO}$ abundance and excitation variations have been studied by searching for evolutionary trends in different samples (Codella et al. 1999; Miettinen et al. 2006; Motte et al. 2007; Sakai et al. 2010; Gerner et al. 2014; Csengeri et al. 2016; Li et al. 2019a; Liu et al. 2020c). Moreover, Jiménez-Serra et al. (2010), Duarte-Cabral et al. (2014), Louvet et al. (2016), and Cosentino et al. (2020) investigated the mechanisms responsible for the broad and narrow line profile of $\mathrm{SiO}$. However, previous studies have been focused on either individual sources through interferometer arrays or large samples with single-dish observations. The origin of the $\mathrm{SiO}$ emission is still an enigma for astronomers without a high resolution survey of $\mathrm{SiO}$ emission toward a large sample of sources with interferometers.

The ALMA Three-millimeter Observations of Massive Starforming regions (ATOMS) survey employed the Band 3 and covered eight spectral windows (two wide spectral windows in upper side band and six spectral windows in lower side band) toward 146 massive star-forming sources (Liu et al. 2020a). The survey provides us larger samples and high resolution to systemically study various molecular gas involved in star formation. In this paper, we statistically analyze $\mathrm{SiO}$ emission of 146 sources using the Atacama Compact 7 $\mathrm{m}$ Array observations (ACA) to better understand shocked gas under different conditions and in different evolutionary trends. We establish a catalogue of the $\mathrm{SiO}$ clumps for future studies. We present the level of shock activities and estimate the fraction of the shocked gas in the clumps. The paper is organized as follows: the sample selection and observations are presented in Sect. 2. Sect. 3 gives our results. We put discussion in Sect. 4 and summary in Sect. 5.

\section{OBSERVATIONS}

\subsection{Sample}

A sample of 146 massive SFRs from the ATOMS survey were investigated in this paper. The sample sources were selected from the CS $J=2-1$ line survey of UC HII regions in the Galactic Plane (Bronfman et al. 1996). The sample sources are complete for protoclusters characterized by bright CS $J=2-1$ emission $\left(T_{\mathrm{b}}>2 \mathrm{~K}\right)$, implying reasonably dense gas. The basic parameters of the sample were taken from Liu et al. (2020a), Liu et al. (2020b), and Liu et al. (2021b) ( paper I, paper II, and paper III of the series). The distance of the sample sources ranges from $0.4 \mathrm{kpc}$ to $13.0 \mathrm{kpc}$ with a mean value of $4.5 \mathrm{kpc}$. The clump mass ranges from 5.6 to $2.5 \times 10^{5} M_{\odot}$, with a median value of $1.4 \times 10^{3} M_{\odot}$. The bolometric luminosity ranges from 16 to $8.1 \times 10^{6} L_{\odot}$, with a median value of $5.7 \times 10^{4} L_{\odot}$. 
(A)

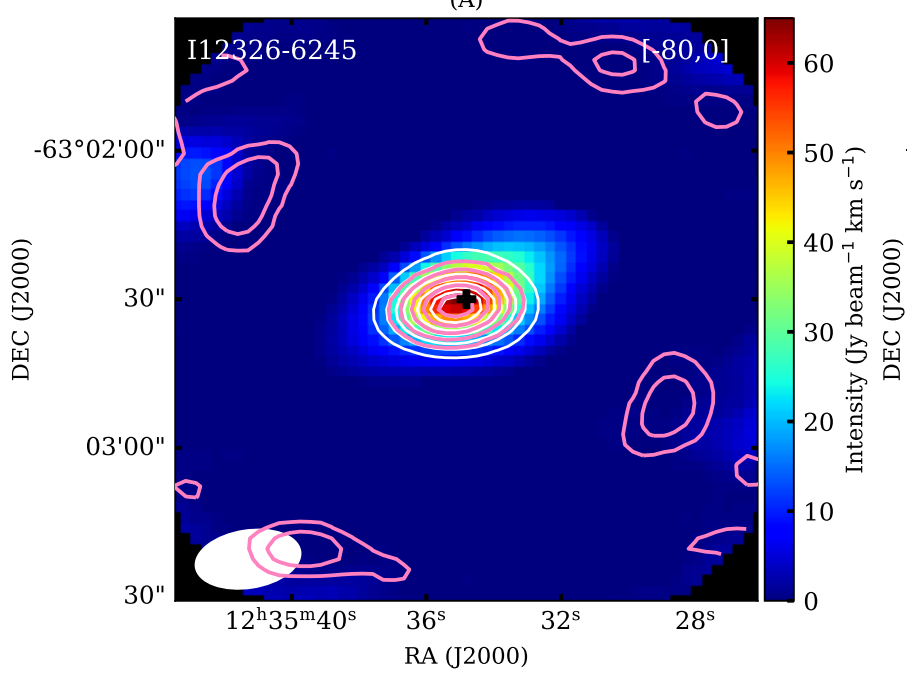

(C)

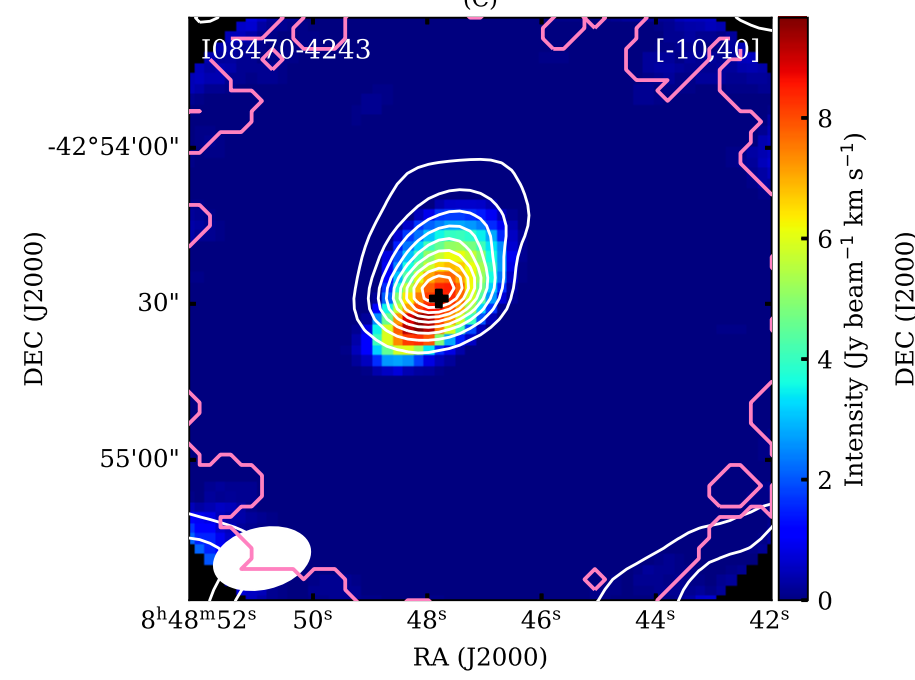

(B)

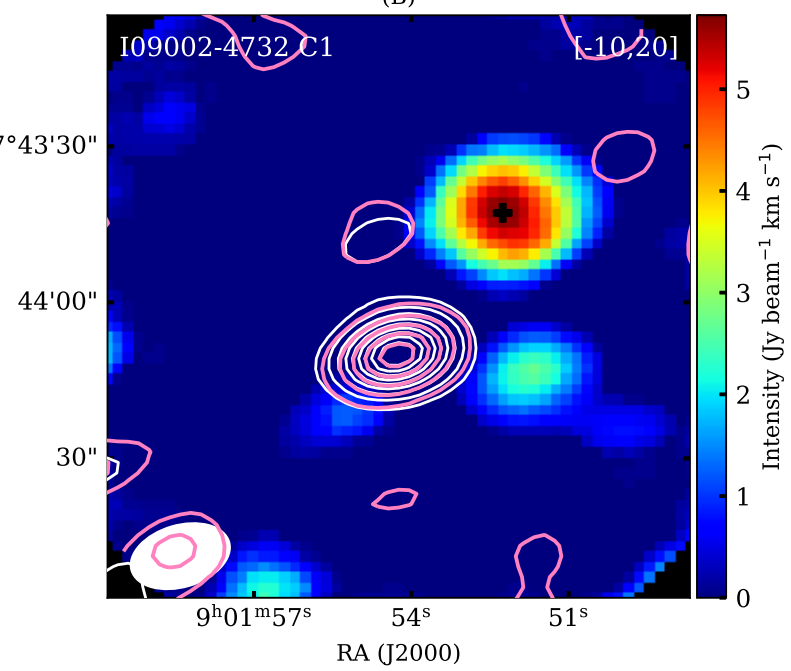

(D)

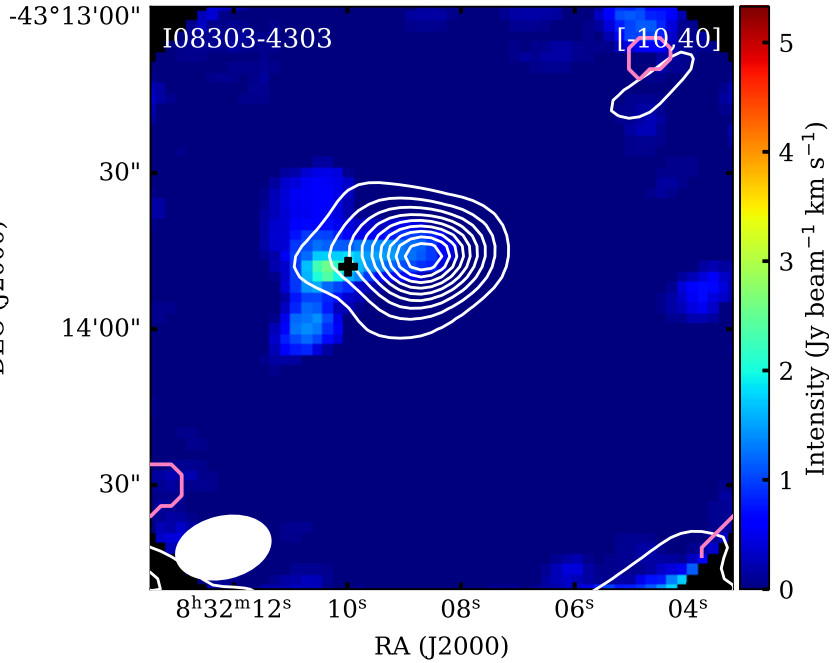

Figure 1. Example sources. The background is $\mathrm{SiO}(2-1)$ integrated intensity maps. The white contours are 3 mm continuum emission, and contours are from $10 \%$ to $100 \%$ in the step of $10 \%$ of peak values. The pink contours represent $\mathrm{H} 40 \alpha$ emission, contours from $10 \%$ to $100 \%$ in the step of $20 \%$ of peak values. The source name is shown on the upper left. The black cross is the central position extracted spectra and the beam size is presented at the lower left corner. The integration ranges are shown in the upper right corner. (A) Panel presents source associated with $3 \mathrm{~mm}$ emission and coincided with H40 $\alpha$ emission. (B) Panel shows source separated with $3 \mathrm{~mm}$ emission and coincided with $\mathrm{H} 40 \alpha$ emission. (C) Panel presents source associated with $3 \mathrm{~mm}$ emission undetected $\mathrm{H} 40 \alpha$ emission. (D) Panel shows source separated with $3 \mathrm{~mm}$ emission undetected $\mathrm{H} 40 \alpha$ emission. All images have a field of view of $2^{\prime} \times 2^{\prime}$.

The radii ranges from 0.06 to $4.26 \mathrm{pc}$, with a median value of $0.86 \mathrm{pc}$. The dust temperature ranges from 18 to $46 \mathrm{~K}$, with a median value of $29 \mathrm{~K}$.

\subsection{ALMA Observations}

The present study is based on the ATOMS survey data (Project ID: 2019.1.00685.S; PI: Tie Liu). The survey has collected both $12 \mathrm{~m}-$ array and ACA data. We focus the latter in this work with an aim at compiling a complete catalog of $\mathrm{SiO}$ emission clumps owing to the benefit from the large field of view of the ACA observations. The Atacama Compact $7 \mathrm{~m}$ Array (ACA) observations were conducted 
(A)

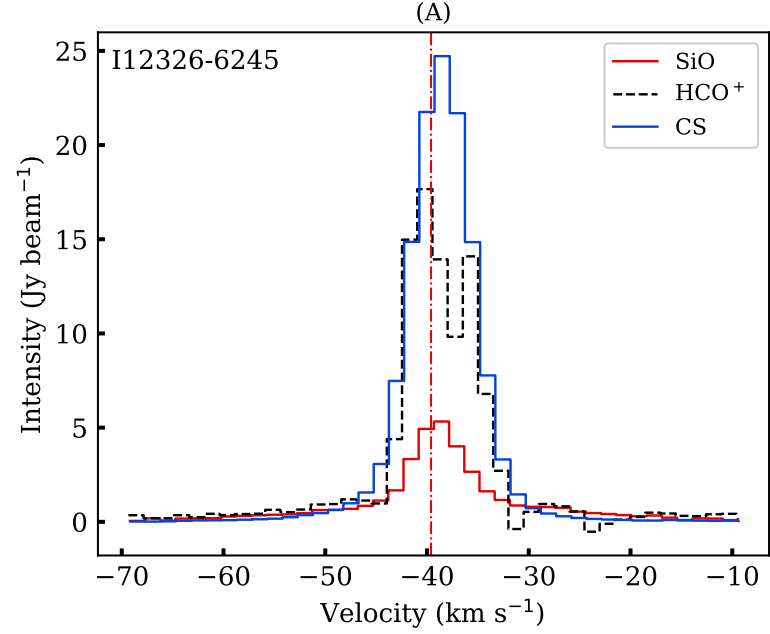

(C)

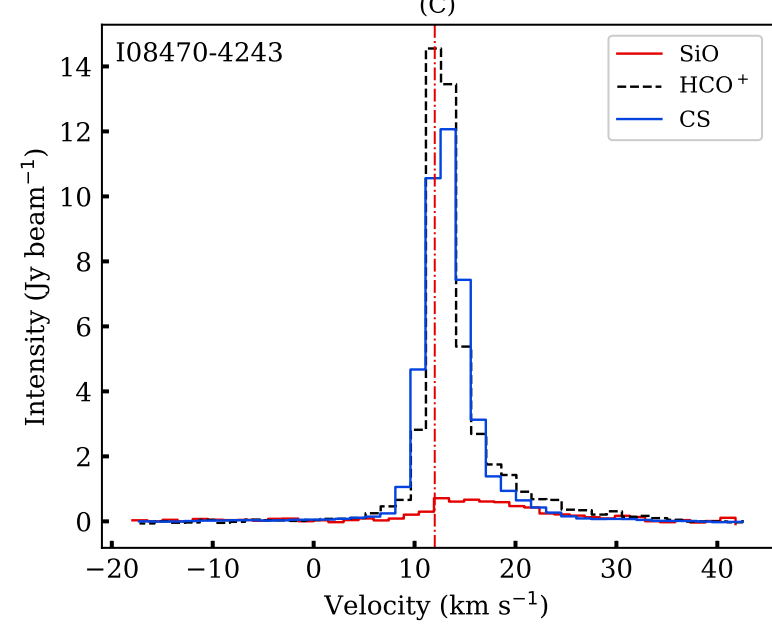

(B)

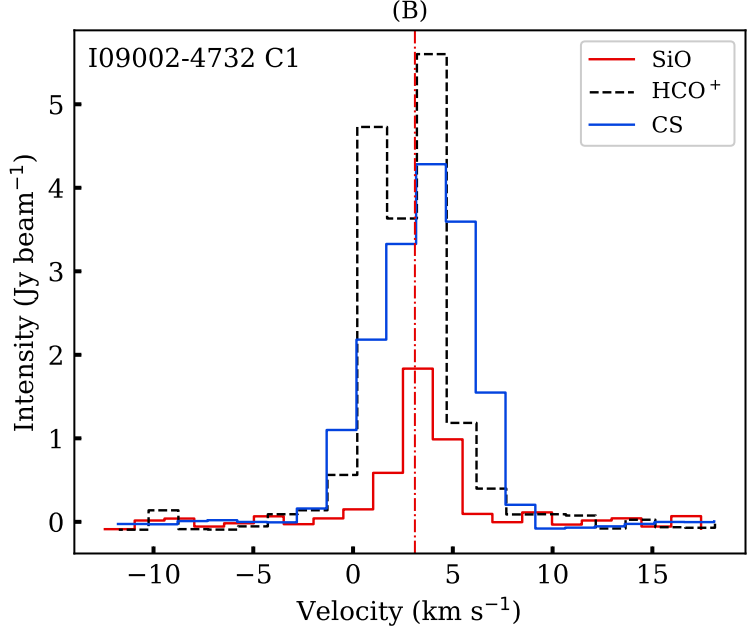

(D)

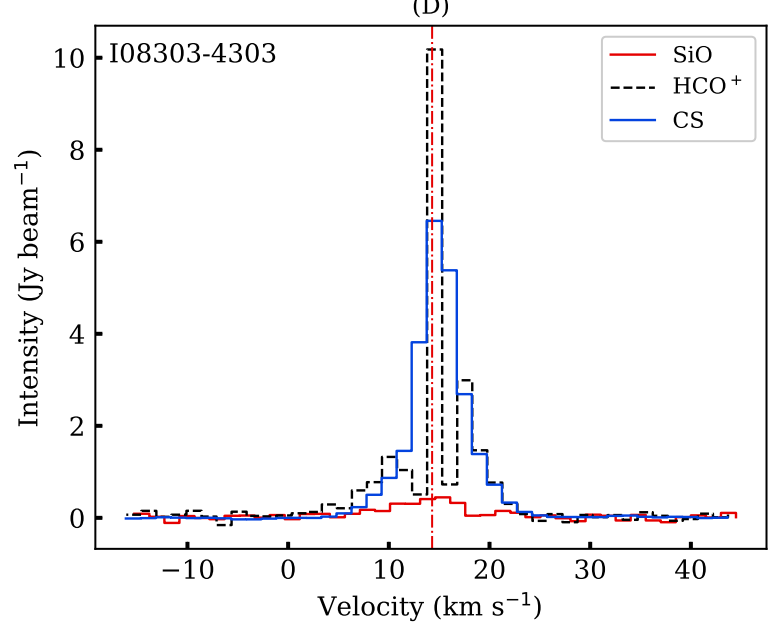

Figure 2. Examples of sources. The red spectrum represents the $\mathrm{SiO}$ average spectral extracted over the aperture of $\mathrm{SiO}$ clumps or beam. The $\mathrm{HCO}^{+}$and $\mathrm{CS}^{-}$ spectra are presented as black dashed and blue solid lines. The dash-dot line indicates the systemic velocity of the source.

from September to mid-November 2019. The typical ACA observing time is $\sim 8$ minutes. The angular resolution of ACA observations is $\sim 13.1^{\prime \prime}-13.8^{\prime \prime}$ and the maximum recovered angular scale is $\sim$ $53.8^{\prime \prime}-76.2^{\prime \prime}$. The $\mathrm{SiO} \mathrm{J}=2-1, \mathrm{HCO}^{+} \mathrm{J}=1-0, \mathrm{H}^{13} \mathrm{CO}^{+} J=1-0$ lines are included in SPWs $1 \sim 6$ at the lower side band with the spectral resolutions of $0.21,0.11$ and $0.21 \mathrm{~km} \mathrm{~s}^{-1}$, respectively (Liu et al. 2021b; Liu et al. 2021a). The H40 $\alpha$ and CS $J=2-1$ lines are included in SPWs 7 at the upper side band with the spectral resolutions of 1.49 $\mathrm{km} \mathrm{s}^{-1}$. The ACA data were calibrated and imaged by CASA software package version 5.6 (McMullin et al. 2007), and more details about data reduction can be found in Paper I.

\section{RESULTS}

For the identification of $\mathrm{SiO}$ clumps, we firstly generate the integrated intensity (Moment 0) maps of $\mathrm{SiO}$ emission using channels with signals higher than $3 \sigma$. The rms level $\sigma$ for each source are listed in Table A1 and Table A2. In Moment-0 maps, we have identified 171 $\mathrm{SiO}$ clumps in 128 sources by eye. We identified $\mathrm{SiO}$ clumps by eye because there are not so many $\mathrm{SiO}$ clumps within individual sources and the emission peaks of most $\mathrm{SiO}$ clumps are clearly separated from each other in these low resolution ACA images. Therefore, we did not apply any algorithm in clump identification in order to avoid fake detection close to the edges of images where the signal-to-noise levels are low. Then we used the two-dimensional fitting tools ( $\mathrm{im} \mathrm{fit}$ ) in CASA to fit these $\mathrm{SiO}$ clumps one by one. We get deconvolved sizes (the full width at half maximum (FWHM) and position angle (PA)), center positions and integrated intensity for these SiO clumps. $\mathrm{SiO}$ clumps in the marginal region are excluded. These results are shown in Table A1 and Table A2. Next we extract the spectra of $\mathrm{SiO}$ lines toward the peak positions of $\mathrm{SiO}$ clumps in all detected sources. The spectra were extracted over the aperture of clumps size or beam size (clumps smaller than the beam size). Examples of the Moment-0 maps and spectra are shown in Appendix A1.

Figure 1 shows four examples of detected sources. A Moment0 map of $\mathrm{SiO}$ emission overlaid by contours of $3 \mathrm{~mm}$ continuum emission and $\mathrm{H} 40 \alpha$ emission is shown. We classify the sources into four groups based on their different conditions in line emission. The classification criteria are as follows:

(A) $27 \mathrm{SiO}$ clumps in sources containing Hı regions: $\mathrm{SiO}$ emission associated with both $\mathrm{H} 40 \alpha$ emission and $3 \mathrm{~mm}$ continuum emission. 
(B) $59 \mathrm{SiO}$ clumps in sources containing Hı regions: $\mathrm{SiO}$ emission separated from both $\mathrm{H} 40 \alpha$ emission and $3 \mathrm{~mm}$ continuum emission.

(C) $39 \mathrm{SiO}$ clumps in sources containing non HII regions: $\mathrm{SiO}$ emission associated with $3 \mathrm{~mm}$ continuum emission.

(D) $46 \mathrm{SiO}$ clumps in sources containing non Hir regions: $\mathrm{SiO}$ emission partly separated from $3 \mathrm{~mm}$ continuum emission.

Figure 2 present the $\mathrm{SiO}(2-1), \mathrm{HCO}^{+}(1-0)$, and CS (2-1) spectra extracted toward four exemplar $\mathrm{SiO}$ clumps. We smooth the spectral resolution of $\mathrm{SiO}$ and $\mathrm{HCO}^{+}$to the spectral resolution of CS $\left(1.49 \mathrm{~km} \mathrm{~s}^{-1}\right)$ for comparison. In I12326-6245, I08470-4243, and I08303-4303, the spectra of SiO show high-velocity wings, and we can see two distinct components: a narrow component and a broad component. The broad $\mathrm{SiO}$ components especially with red- and/or blue-shifted line wings are likely related to molecular outflows. In I12326-6245, I08470-4243, and I08303-4303, the spectra of $\mathrm{HCO}^{+}$ and CS also show high-velocity wings. In I09002-4732 C1, there is no high-velocity wing emission in all lines, indicating that this source does not have outflows. The spectra of other SiO clumps are shown in Appendix A1.

In most detected $\mathrm{SiO}$ clumps, we can see the peak velocity of the narrow components is similar to the systemic velocity (marked by the red dash-dot line). We thus consider these components coincided with the ambient cloud. In very rare cases such as I08076-3556 (Appendix A1), however, the $\mathrm{SiO}$ velocity $\left(\sim 40 \mathrm{~km} \mathrm{~s}^{-1}\right)$ deviated clearly from the systemic velocity $\left(5.9 \mathrm{~km} \mathrm{~s}^{-1}\right)$ and $\mathrm{SiO}$ line emission mainly comes from red-shifted line wings.

\subsection{Detection rates of $\mathrm{SiO}$ emission}

Toward the 146 sources, $\mathrm{SiO}$ (2-1) emission was detected in 128 $(87.7 \%)$ sources, indicating that the presence of shocks is very common in high-mass star forming regions. In particular, when we inspect the spectra of the sources that do not show strong $\mathrm{SiO}$ emission in their Moment- 0 maps, we find additional $18 \mathrm{SiO}$ clumps showing weak $\mathrm{SiO}$ emission, with peak intensity close to $2 \sigma$. We list these weak SiO clumps in Table A2.

Because of the complicated and non-Gaussian SiO line profiles, we instead use their Moment- 0 maps, the intensity-weighted velocity (Moment 1) maps, and the intensity-weighted velocity dispersion (Moment 2) maps to derive their velocity $(v)$, and velocity dispersion $\left(\sigma_{\mathrm{V}}\right)$. The derived parameters of $\mathrm{SiO}$ clumps are summarized in Table A1. Notably, the velocity dispersion of $\mathrm{SiO}$ emission is mostly larger than the velocity resolution $\left(0.21 \mathrm{~km} \mathrm{~s}^{-1}\right)$. For the 18 weaker clumps, we use one component Gaussian fit of their averaged spectra to get the line parameters (Intensity, velocity, and velocity dispersion). The derived line parameters are shown in Table A2.

\subsection{The velocity dispersion of $\mathrm{SiO}$ and the line wings of $\mathrm{SiO}$, $\mathrm{HCO}^{+}$, and $\mathrm{CS}$}

The velocity dispersions of the detected $\mathrm{SiO}$ lines are listed in Table $\mathrm{A} 1$ and Table A2. For the $\mathrm{SiO}$ lines, the range of the velocity dispersion values is $0.3 \sim 5.43 \mathrm{~km} \mathrm{~s}^{-1}$. The mean values is $1.77 \mathrm{~km}$ $\mathrm{s}^{-1}$, and the median values is $1.59 \mathrm{~km} \mathrm{~s}^{-1}$. The histogram of the velocity dispersion is presented in Figure 3.

To identify strong outflows in these sources, we searched the line wings in $\mathrm{SiO}, \mathrm{HCO}^{+}$, and $\mathrm{CS}$ emission lines. These results are listed in Table A3. Because of relatively high abundance, $\mathrm{HCO}^{+}$emission is a very good tracer of outflows (Myers et al. 1996). Therefore, in outflow regions, $\mathrm{HCO}^{+}$emission is detectable even if $\mathrm{SiO}$ emission is not detected. However, in some sources the line profile of $\mathrm{HCO}^{+}$

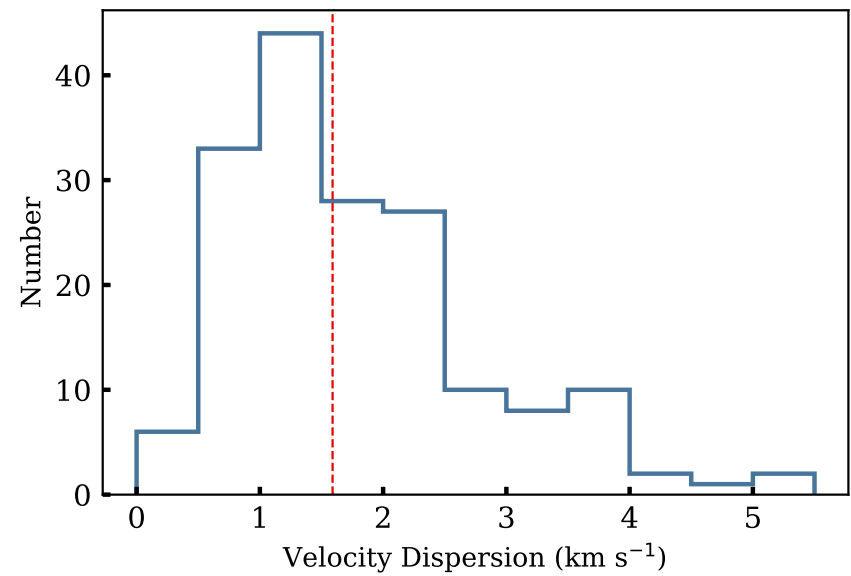

Figure 3. Histogram of the velocity dispersion of the $\mathrm{SiO}(2-1)$ lines. The vertical red dashed line represents the median velocity dispersion.

emission is self-absorbed. We inspect the spectra of CS and find its spectra to be less self-absorbed. As a consequence, we use the line wings of $\mathrm{SiO}, \mathrm{HCO}^{+}$, and $\mathrm{CS}$ together to identify strong outflows. The spectra of $\mathrm{SiO}, \mathrm{HCO}^{+}$, and $\mathrm{CS}$ are shown in Appendix A1. According to the high velocity line wings, the $\mathrm{SiO}$ clumps are divided into two groups: clumps with strong outflows and without strong outflows. We find 116 clumps are associated with outflows showing wing emission in at least one of the three lines and 50 clumps are not associated with outflows. However, we cannot exclude these clumps without outflows that are not associated with weak outflows. We exclude I17441-2822 spectra in further analysis because this source is close to the galactic center and shows very complicated line profiles.

\section{3 $\mathrm{SiO}$ clumps properties}

Using the Moment-0 maps of $\mathrm{SiO}$ emission, we have identified 171 $\mathrm{SiO}$ clumps. We calculated the geometric mean of the major axis FWHM and the minor axis FWHM and got the SiO clumps linear radius. The histogram of the $\mathrm{SiO}$ clump radii is presented in Figure 4. The range of the $\mathrm{SiO}$ clump radii is $0.03 \sim 0.99 \mathrm{pc}$. The mean value is $0.3 \mathrm{pc}$, and the median value is $0.23 \mathrm{pc}$. In addition, we failed to fit 116 clumps because the shape of $\mathrm{SiO}$ clumps is irregular and the size of these $\mathrm{SiO}$ clumps is smaller than or close to the beam size. Instead, we give the beam size as these clump sizes, and we marked these clumps in Table A1. The velocities of SiO clumps are approximately consistent with the systemic velocities of the clumps, except for I08076-3556 (Sect. 3).

In Moment- 0 maps, we found that $105 \mathrm{SiO}$ clumps are not spatially coincided with the peak of $3 \mathrm{~mm}$ continuum emission with separation larger than half of beam size. There are $66 \mathrm{SiO}$ clumps associated with $3 \mathrm{~mm}$ continuum emission. We derived the distances between the central positions of $\mathrm{SiO}$ clumps and the peak positions of the 3 $\mathrm{mm}$ continuum emission. These results are listed in Table A3. The histogram of the separation distance is presented in Figure 4. The range of the separation is $0 \sim 3.35 \mathrm{pc}$. The mean value is $0.3 \mathrm{pc}$, and the median value is $0.17 \mathrm{pc}$. An evident tail is presented in the separation histogram which represents these $\mathrm{SiO}$ clumps away from the $3 \mathrm{~mm}$ continuum emission may not be caused by outflows. This separation between the thermal dust and $\mathrm{SiO}$ molecular gas is similar to the results of López-Sepulcre et al. (2016) and Li et al. (2020).

For the 128 sources with $\mathrm{SiO}$ emission, 63 sources have $\mathrm{H} 40 \alpha$ 
(a)

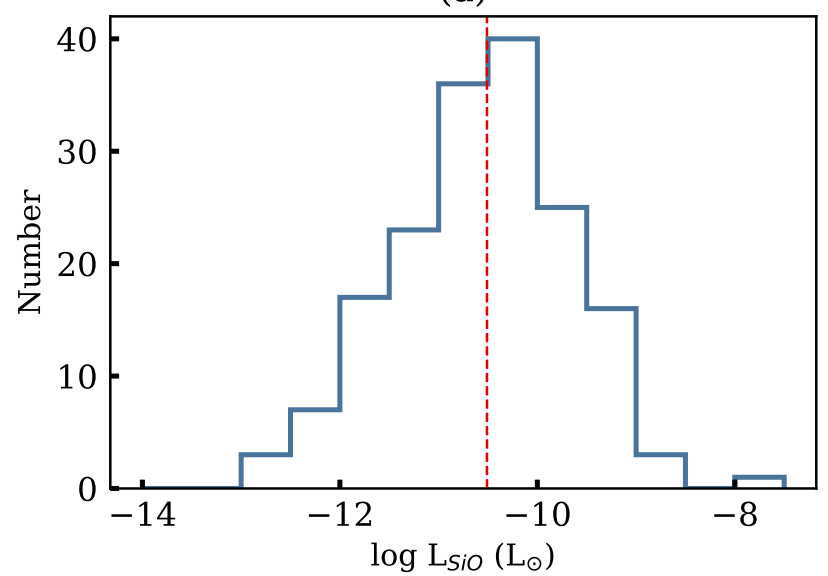

(c)

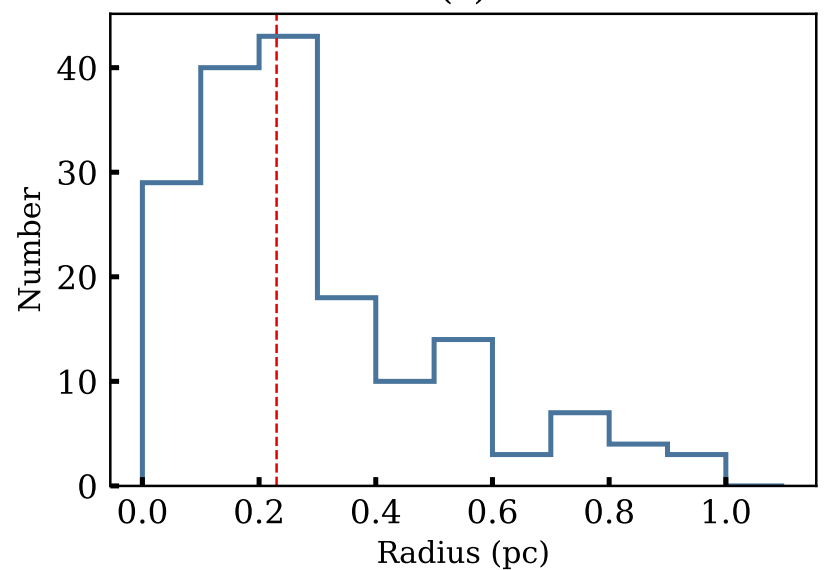

(b)

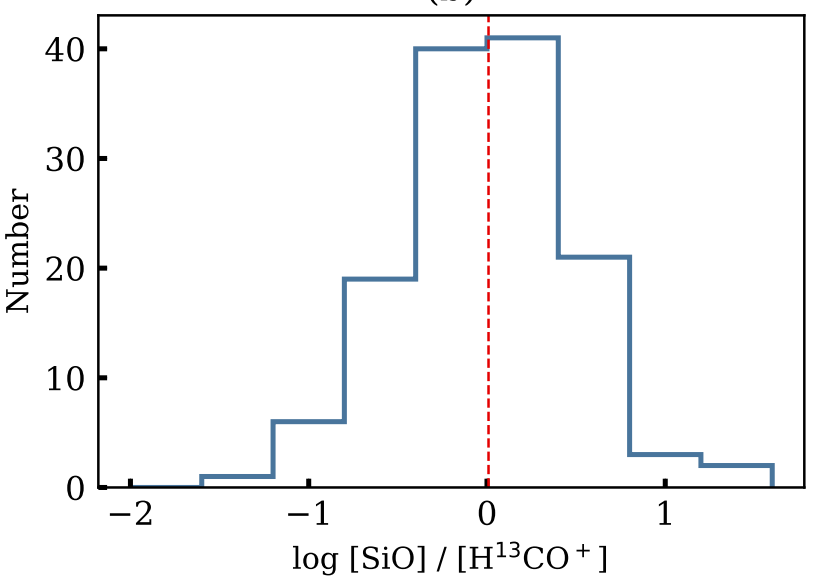

(d)

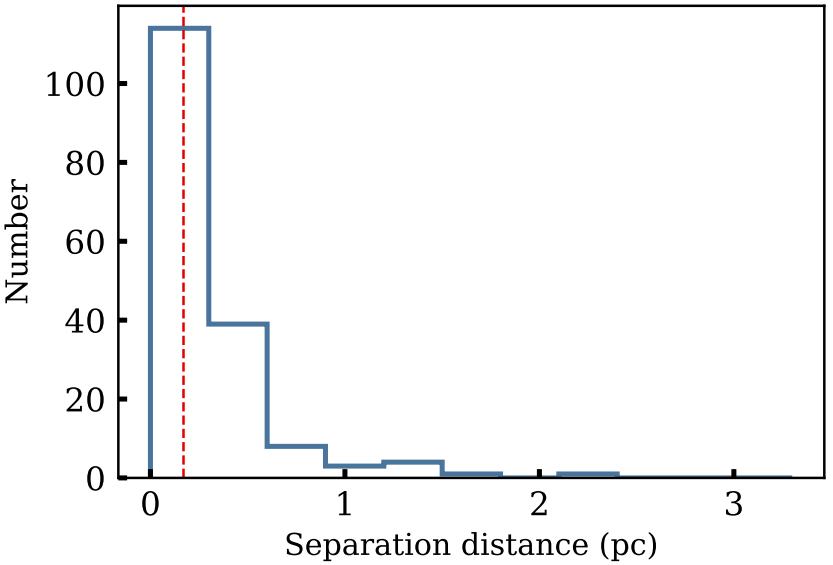

Figure 4. (a) Histogram of the $\mathrm{SiO}$ luminosity. (b) Histogram of the $[\mathrm{SiO}] /\left[\mathrm{H}^{13} \mathrm{CO}^{+}\right]$ratio. (c) Histogram of separation distance. (d) Histogram of the SiO clump radius. The vertical red dashed line represents the median values of the parameter.

emission above $3 \sigma$. The $\mathrm{H} 40 \alpha$ emission shows a compact structure and is coincident with the $3 \mathrm{~mm}$ continuum emission, indicating the existence of compact Hir regions. For some of these sources, we find that $\mathrm{SiO}$ clumps are surrounding the $\mathrm{H} 40 \alpha$ emission, indicating the possible existence of shocks induced by interaction between HII regions and their surrounding molecular clouds (Cosentino et al. 2020; Liu et al. 2020a). We will investigate the properties of shocked gas caused by Hı regions using the high resolution 12-m array data in a forthcoming work.

We calculate the $\mathrm{SiO}(2-1)$ luminosity $\left(L_{\mathrm{SiO}}\right)$ of the $\mathrm{SiO}$ clumps using the integrated intensity and the source distance. $L_{\mathrm{SiO}}$ can be derived from the formula:

$L_{\mathrm{SiO}}=4 \pi \times d^{2} \times \int F_{\nu} d v$

where $\mathrm{d}$ is the distance to the source and $v$ represents the velocity. $\int F_{v} d v$ is the integrated integrated intensity of the SiO clump.

$L_{\mathrm{SiO}}$ from $1.74 \times 10^{-13}$ to $1.07 \times 10^{-8} L_{\odot}$. The mean value is 2.69 $\times 10^{-11} L_{\odot}$, and the median value is $3.1 \times 10^{-11} L_{\odot}$. Figure 4 (a) shows the number distribution of the $\mathrm{SiO}$ luminosity. We used the sum $L_{\mathrm{SiO}}$ in one source for statistic analysis. More details on $L_{\mathrm{SiO}}$ will be discussed in Sect. 4.2.

\subsection{The fraction of shocked gas}

The $\mathrm{H}^{13} \mathrm{CO}^{+}$emission is a good tracer of relatively quiescent gas. The $\mathrm{H}^{13} \mathrm{CO}^{+}$abundance does not vary substantially with time (Nomura \& Millar 2004). Therefore, column density of $\mathrm{H}^{13} \mathrm{CO}^{+}$ could reflect the dense gas of the clump. Sakai et al. (2010) used $\mathrm{SiO}$ column density against $\mathrm{H}^{13} \mathrm{CO}^{+}$column density to represent the fraction of shocked gas in a dense clump. Assuming both $\mathrm{SiO}$ and $\mathrm{H}^{13} \mathrm{CO}^{+}$emission are optically thin and have constant excitation temperatures, the integrated intensity ratios of $\mathrm{SiO}$ emission and $\mathrm{H}^{13} \mathrm{CO}^{+}$emission can approximately reflect the relative fraction of the shocked gas in the clump, and later we use $[\mathrm{SiO}] /\left[\mathrm{H}^{13} \mathrm{CO}^{+}\right]$ to represent this ratio. The derived ratios are shown in Table A3. We inspected $\mathrm{H}^{13} \mathrm{CO}^{+}$spectra and found that 12 sources have absorption features in their spectra. As a result, in the latter analysis, we ignore these 12 sources. For sources containing multiple $\mathrm{SiO}$ 
(a)

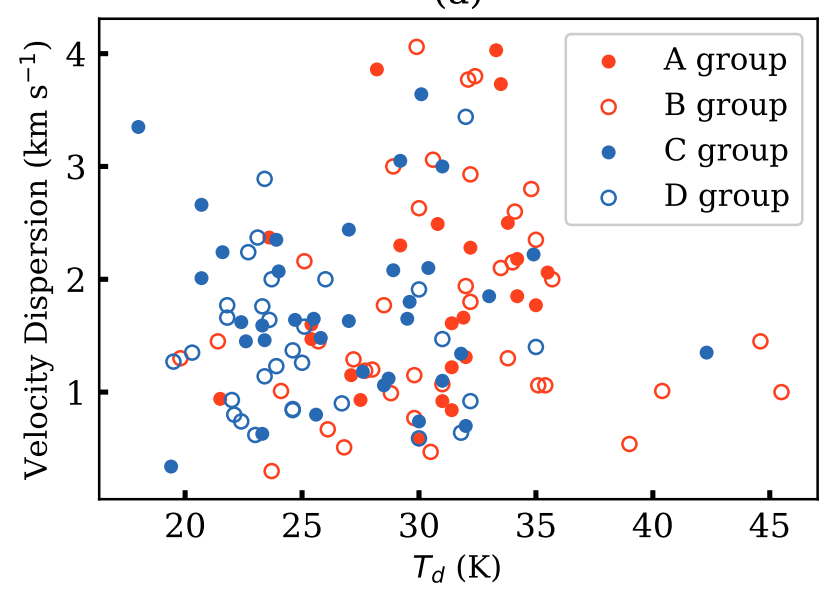

(c)

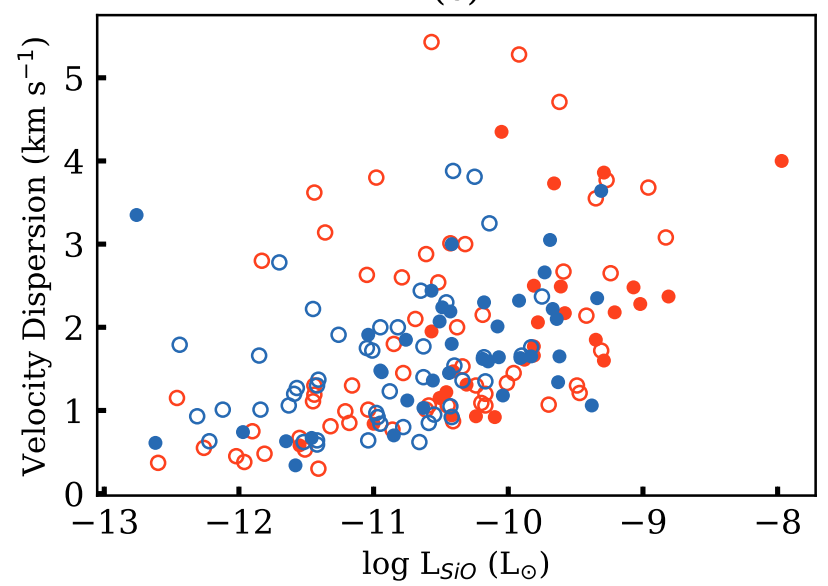

(b)

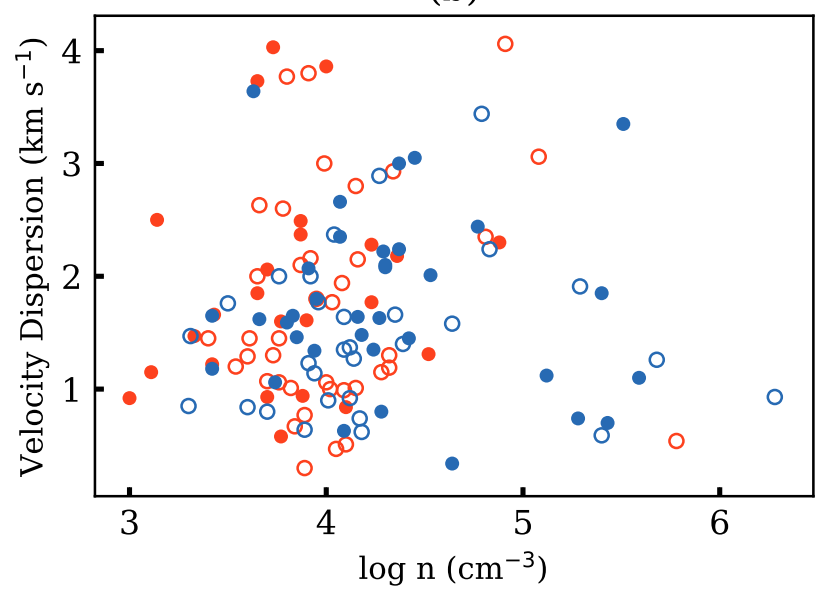

(d)

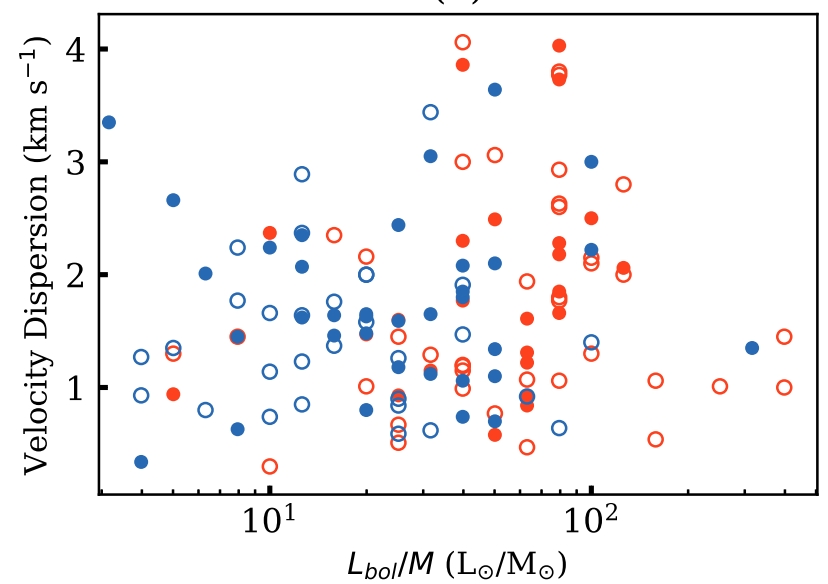

Figure 5. (a) The velocity dispersion of $\mathrm{SiO}$ emission against dust temperature $\left(T_{\mathrm{d}}\right)$. (b) The velocity dispersion of $\mathrm{SiO}$ emission versus the particle number density $(n)$ of the clumps. (c) The velocity dispersion of $\mathrm{SiO}$ emission against the $\mathrm{SiO}$ clump radius. (d) The velocity dispersion of $\mathrm{SiO}$ emission vs. the bolometric luminosity to mass ratio $\left(L_{\mathrm{bol}} / M\right)$. The filled red circles (A group) represent the $\mathrm{SiO}$ clumps containing HiI regions are associated with 3 mm continuum emission. The empty red circles (B group) represent the SiO clumps containing Hir regions are separated with 3 mm continuum emission. The filled blue circles ( $\mathrm{C}$ group) represent the $\mathrm{SiO}$ clumps containing non HII regions are associated with $3 \mathrm{~mm}$ continuum emission. The empty blue circles (D group) represent the $\mathrm{SiO}$ clumps containing non $\mathrm{HII}$ regions are separated with $3 \mathrm{~mm}$ continuum emission.

clumps, we calculate an average $[\mathrm{SiO}] /\left[\mathrm{H}^{13} \mathrm{CO}^{+}\right]$ratio. The range of $[\mathrm{SiO}] /\left[\mathrm{H}^{13} \mathrm{CO}^{+}\right]$is $0.04 \sim 42.79$. The mean values is 2.41 , and the median values is 1.02 . The histogram of $[\mathrm{SiO}] /\left[\mathrm{H}^{13} \mathrm{CO}^{+}\right]$of all sources is presented in Figure 4 (b).

\section{DISCUSSION}

\subsection{The line broadening of $\mathrm{SiO}$ emission}

The broad components of $\mathrm{SiO}$ emission in our sample are likely caused by the high-velocity gas driven by energetic outflows. The high detection rate of the velocity wings of $\mathrm{SiO}$ emission $(60 \%)$ suggests the presence of outflows. Our finding divides the $\mathrm{SiO}$ clumps into two groups (Sect. 3.2). In the group with outflows, the median velocity dispersion of the $\mathrm{SiO}$ emission is as large as $1.91 \mathrm{~km} \mathrm{~s}^{-1}$.
In the other group without outflows, the narrow components of $\mathrm{SiO}$ emission are common and the median velocity dispersion of the $\mathrm{SiO}$ emission is $0.99 \mathrm{~km} \mathrm{~s}^{-1}$. This narrow $\mathrm{SiO}$ emission could be created by unresolved low mass outflows, cloud-cloud collision, or gas inflows.

We plot the cumulative distribution of the $\mathrm{SiO}$ velocity dispersion for these two groups in Figure 6. The SiO clumps with outflows are presented as red curve, while the clumps without outflows are shown as blue curve. For the $\mathrm{SiO}$ clumps with strong outflows, the velocity dispersion values range from 0.58 to $5.43 \mathrm{~km} \mathrm{~s}^{-1}$ with a mean value of $2.08 \mathrm{~km} \mathrm{~s}^{-1}$ and a standard deviation of $1.00 \mathrm{~km} \mathrm{~s}^{-1}$. In contrast, for the $\mathrm{SiO}$ clumps without strong outflows, the velocity dispersion values are from 0.32 to $3 \mathrm{~km} \mathrm{~s}^{-1}$, with a mean value of $1.06 \mathrm{~km}$ $\mathrm{s}^{-1}$ and a standard deviation of $0.53 \mathrm{~km} \mathrm{~s}^{-1}$. In addition, we use the Kolmogorov-Smirnov (KS) test to compare these distributions. The 


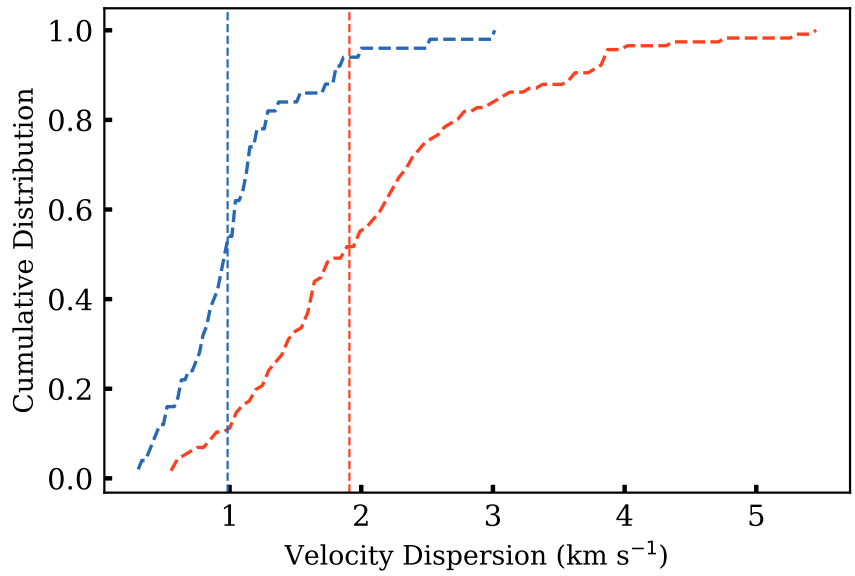

Figure 6. The velocity dispersion distributions for $\mathrm{SiO}$ clumps with outflows and without outflows (the red and blue lines). The vertical dashed lines represent the median velocity dispersion of $\mathrm{SiO}$ clumps in the two groups: $1.91 \mathrm{~km} \mathrm{~s}^{-1}$ (red line) and $0.99 \mathrm{~km} \mathrm{~s}^{-1}$ (blue line) for clumps with and without strong outflows, respectively.

P-value returned by the KS test is the probability that the two samples were drawn from the same distributions. If the P-value is smaller than $5 \%$, we conclude that the two samples were drawn from different distributions. The KS test gives a P-value of about $10^{-12}$, indicating that the velocity dispersion distribution of these two groups is very different. The clumps associated with outflows show significantly larger velocity dispersion in $\mathrm{SiO}$ emission on average.

In shocked gas, the $\mathrm{SiO}$ velocity dispersion is attributed to nonthermal broadening. Gusdorf et al. (2008a) found that the SiO intensity and line width as the shock dissipates. In Figure 5, we plot the velocity dispersion of the four groups (Sect. 3) against dust temperature $\left(T_{\mathrm{d}}\right)$, the particle number density $(n)$ of the clumps, the $\mathrm{SiO}$ luminosity $\left(L_{\mathrm{sio}}\right)$, and the bolometric luminosity to mass ratio $\left(L_{\mathrm{bol}} / M\right)$ of clumps from Liu et al. (2020a), respectively. The particle number density can be derived as

$n=\frac{3 M_{\text {gas }}}{4 \pi R^{3} m_{H} \mu}$,

$M$ and $R$ the clump mass and effective radii compiled in Liu et al. (2020a). $n$ is the particle number density. $m_{\mathrm{H}}$ is the mass of a hydrogen atom. $\mu=2.37$ is the mean molecular weight per "free particle". $T_{\mathrm{d}}$ and $L_{\mathrm{bol}} / M$ are potential evolutionary tracers for high-mass protostars and their natal clumps (Molinari et al. 2008; Elia et al. 2021). More evolved sources show higher $T_{\mathrm{d}}$ and $L_{\mathrm{bol}} / M$.

As shown in Figure 5, there is no obvious trend between the velocity dispersion of all groups $\mathrm{SiO}$ emission and either $T_{\mathrm{d}}$ or $n$. There is also no statistically significant correlation between the velocity dispersion of all groups and $L_{\text {bol }} / M$. We use the Spearman-rank correlation test between those quantities, and the correlation coefficient is $0.15,0.06$, and 0.13 , respectively. In our samples, we found no obvious variations in the velocity dispersion of the $\mathrm{SiO}$ emission against $T_{\mathrm{d}}, n$, and $L_{\mathrm{bol}} / M$, which indicates that the strengths of shocks are not so different under different physical conditions or at various evolutionary stages. Our results are consistent with the results of Csengeri et al. (2016) and Li et al. (2019a), who also found the line width of $\mathrm{SiO}$ emission is nearly constant at different evolutionary stages of clumps. In A, B, C groups, the $L_{\text {sio }}$ has a positive correlation with the velocity dispersion of $\mathrm{SiO}$ emission, with a cor-
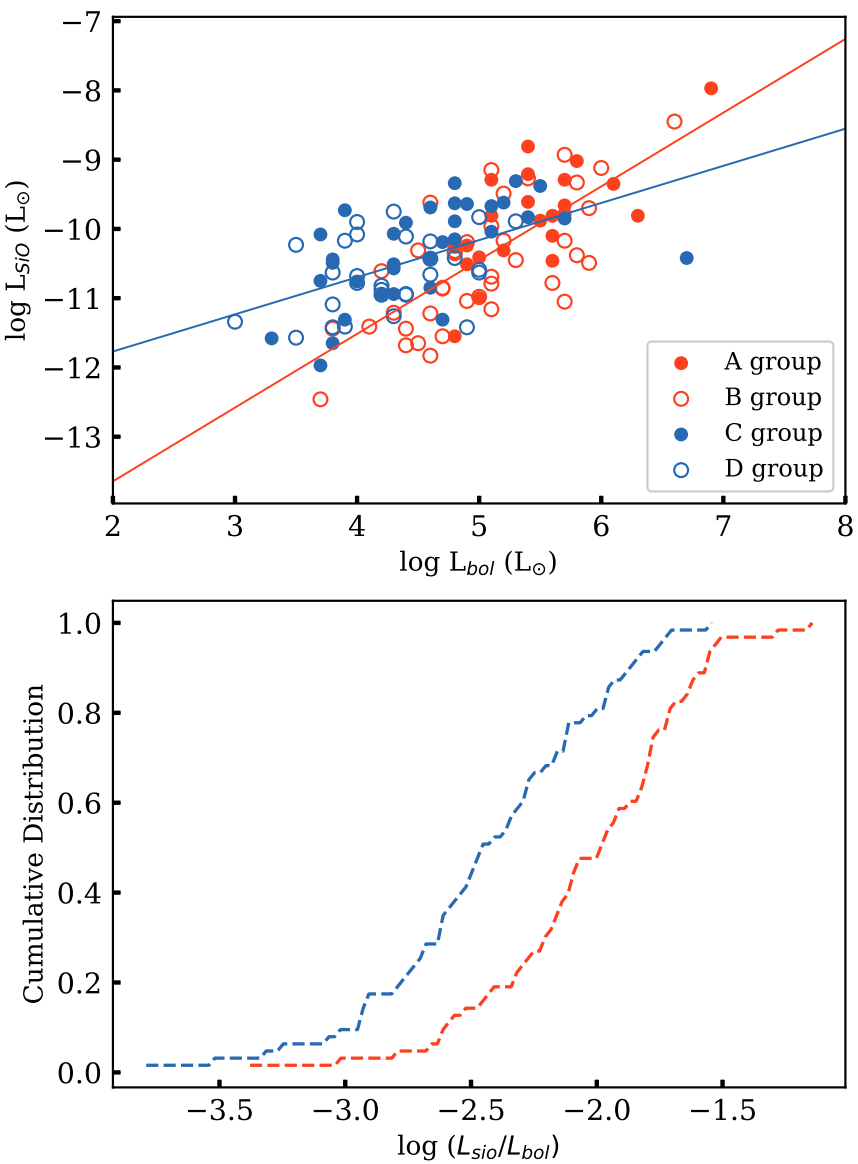

Figure 7. Upper panel: $\mathrm{SiO}(2-1)$ line luminosity $L_{\mathrm{SiO}}$ versus bolometric luminosity $L_{\mathrm{bol}}$. The red line shows a linear fit of $f(x)=(1.06 \pm 0.12) x-$ 15.77 and the blue line a linear fit of $f(x)=(0.54 \pm 0.11) x-12.84$. The symbols are the same with Figure 5. Lower panel: The $L_{\mathrm{SiO}} / L_{\text {bol }}$ distributions for $\mathrm{SiO}$ clumps with $\mathrm{H}_{\text {II }}$ regions and without $\mathrm{H}_{\text {II }}$ regions (the red and blue lines).

relation coefficient of $0.72,0.55$, and 0.44 , respectively. These results suggest that the more intense $\mathrm{SiO}$ sources are associated with more active outflows. Whereas in the D group, the $L_{\text {sio }}$ does not correlate with the velocity dispersion of $\mathrm{SiO}$ emission. It means it presence of relatively strong $\mathrm{SiO}$ emission sources has smaller velocity dispersion. One would expect these $\mathrm{SiO}$ clumps may be due to the aging of outflows as it moves away from its driving source. An alternative explanation is to consider some of these $\mathrm{SiO}$ sources caused by unresolved lower mass protostars, cloud-cloud collision, or gas inflows. We will investigate these scenarios in the following work with high resolution 12-m array data.

\subsection{The excitation condition for $\mathrm{SiO}$ emission}

We examine the relationship between the bolometric luminosity and $\mathrm{SiO}$ emission line luminosity in all groups. Figure 7 shows the $\mathrm{SiO}$ line luminosity versus the bolometric luminosity in the upper panel. 

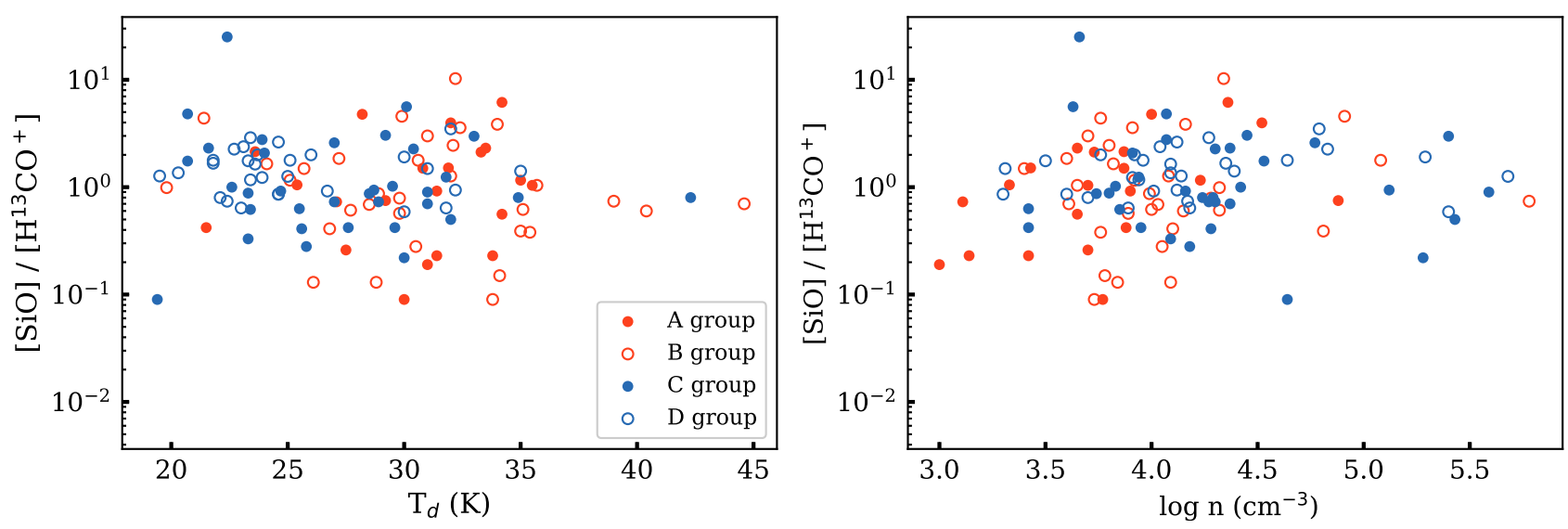

Figure 8. Left: $[\mathrm{SiO}] /\left[\mathrm{H}^{13} \mathrm{CO}^{+}\right]$against dust temperature $\left(T_{\mathrm{d}}\right)$. Right: $[\mathrm{SiO}] /\left[\mathrm{H}^{13} \mathrm{CO}^{+}\right]$vs. particle number density $(n)$. The symbols are the same as in Figure 5 .
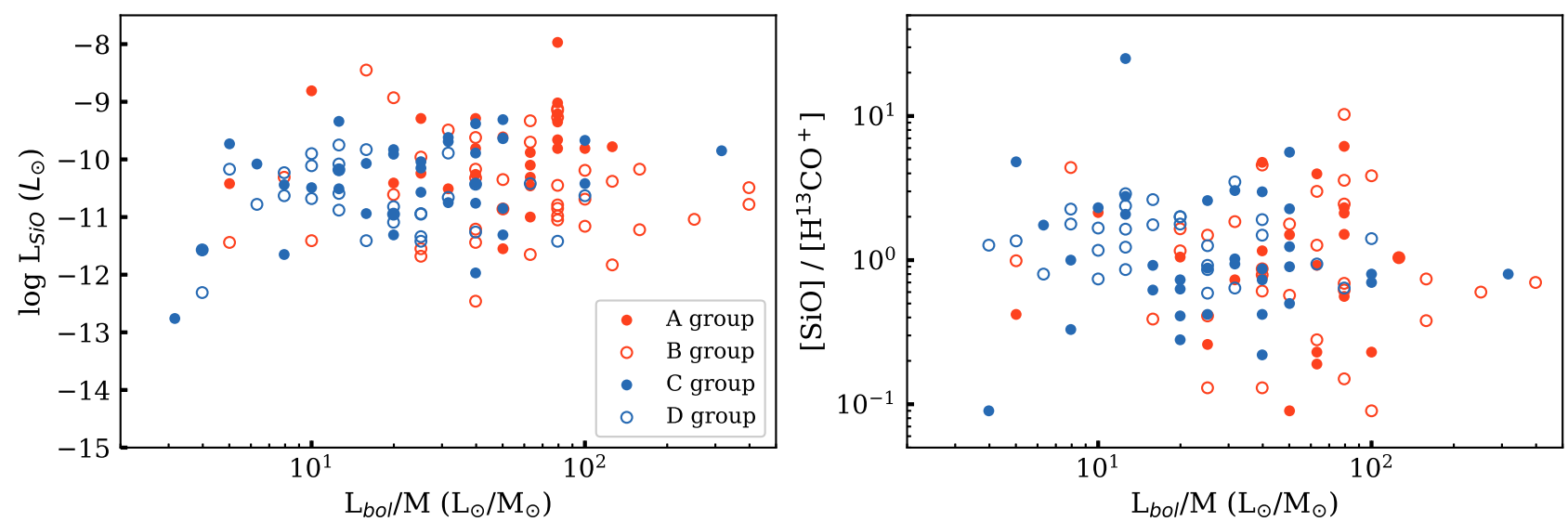

Figure 9. Left: The $\mathrm{SiO}$ line luminosity versus $L_{\mathrm{bol}} / M$ in the clumps. Right: $[\mathrm{SiO}] /\left[\mathrm{H}^{13} \mathrm{CO}^{+}\right]$vs. $L_{\mathrm{bol}} / M$. The symbols are the same with Figure 5

We exclude two low-mass star forming sources (I08076-3556 and I11590-6452; Liu et al. 2020a). We can see an increasing trend between these two quantities in all groups. But in the A and B groups (with Hit regions), the increasing trend is much steeper. Thus we get two linear fits. In the groups with HII regions, a linear fit is $f(x)=(1.06 \pm 0.12) x-15.77$ and in the groups without Hir regions, another linear fit is $f(x)=(0.54 \pm 0.11) x-12.84$. The Spearman rank correlation coefficient (p) is 0.7 and 0.58 , respectively. This implies that higher luminosity sources could have brighter $\mathrm{SiO}$ emission, indicating stronger shock activity in more luminous protoclusters. These results are consistent with the results of Codella et al. (1999) and Liu et al. (2021c), who both found a trend of brighter $\mathrm{SiO}$ emission in higher luminosity sources. As for the different slope of $L_{\text {sio }} / L_{\text {bol }}$, this could be due to the different evolutionary stages of these sources. Next, we plot the cumulative distribution of the $L_{\text {sio }} / L_{\text {bol }}$ for the $\mathrm{SiO}$ clumps with HII regions and without HII regions in the lower panel. The KS test reveals that the p-value is $10^{-5}$, indicating the $L_{\mathrm{sio}} / L_{\mathrm{bol}}$ for these two groups is from different distributions. Sources containing His regions show relatively lower $L_{\text {sio }} / L_{\text {bol }}$ ratios, indicating that $\mathrm{H}_{\mathrm{II}}$ regions may have negative feedback on surrounding gas and may suppress further star formation, leading to less energetic outflows and thus weaker shocks.
Figure 8 shows $[\mathrm{SiO}] /\left[\mathrm{H}^{13} \mathrm{CO}^{+}\right]$intensity ratio as a function of $T_{\mathrm{d}}$ and $n$ in all groups ( $\mathrm{p}=-0.13,0.12$, respectively). However, we find the fraction of shocked gas increase as the $n$ increase in A group $(p=0.5)$. Summarizing, in a large fraction of sources (except for the A group), the fraction of shocked gas shows no essential dependence on the $T_{\mathrm{d}}$ and $n$. This indicates that $\mathrm{SiO}$ emission is not affected by thermal condition but is more likely affected by shock activities. However, $\mathrm{H}^{13} \mathrm{CO}^{+}$abundance may not be constant with time (Sanhueza et al. 2012), which may affect the interpretation of $[\mathrm{SiO}] /\left[\mathrm{H}^{13} \mathrm{CO}^{+}\right]$ratios.

The bolometric luminosity of a molecular clump will increase as the high mass star evolves, while its mass will decrease. Thus the bolometric luminosity to mass ratio $\left(L_{\mathrm{bol}} / M\right)$ can be a good tracer of the evolutionary stage of star formation (Molinari et al. 2008, 2016; Elia et al. 2021). $L_{\mathrm{bol}} / M$ values can distinguish between the young and evolved sources, and its low values are related to young sources. In the left panel of Figure 9, we plot the $\mathrm{SiO}$ luminosity $\left(L_{\mathrm{SiO}}\right)$ against $L_{\mathrm{bol}} / M$ in all groups $(\mathrm{p}=0.3,-0.04,0.32$, and -0.12 , respectively). The $L_{\mathrm{SiO}}$ does not vary with $L_{\mathrm{bol}} / M$, suggesting that SiO luminosity has no relationship with evolutionary stages, which is similar to the results of Liu et al. (2021c). The right panel shows $[\mathrm{SiO}] /\left[\mathrm{H}^{13} \mathrm{CO}^{+}\right]$ versus $L_{\mathrm{bol}} / M$. There is also no correlation between $[\mathrm{SiO}] /\left[\mathrm{H}^{13} \mathrm{CO}^{+}\right]$ 
and $L_{\mathrm{bol}} / M$. This implies that the fraction of shocked gas in highmass star forming clumps does not change obviously in dense gas at various evolutionary stages. This is consistent with the results reported by Csengeri et al. (2016) and Li et al. (2019a).

\section{SUMMARY}

In this work, we used ALMA ACA observational data for a statistical study of shocked gas toward 146 massive star forming regions. We analyze the variation of $\mathrm{SiO}$ emission under different physical conditions and evolutionary stages. The main results are summarized as follows:

(1) Among the entire sample, we have detected $\mathrm{SiO}$ emission in 128 sources, which contain $171 \mathrm{SiO}$ clumps. $\mathrm{SiO}(2-1)$ emission has a high detection rate of $87.7 \%$, above $3 \sigma$.

(2) The velocity dispersion of $\mathrm{SiO}$ line emission ranges from 0.3 to $5.43 \mathrm{~km} \mathrm{~s}^{-1}$, with a median velocity dispersion of $1.59 \mathrm{~km} \mathrm{~s}^{-1}$. Based on the high-velocity emission wings in $\mathrm{SiO}, \mathrm{HCO}^{+}$, and $\mathrm{CS}$ lines, we divided the clumps into two groups. There are $116 \mathrm{SiO}$ clumps associated with strong outflows, which show high-velocity wing emission in at least one line, while the other $50 \mathrm{SiO}$ clumps show no wing emission in the three lines and seem to be not associated with energetic outflows. The two groups have an obvious difference in the velocity dispersion of $\mathrm{SiO}$ emission. The median velocity dispersion of outflow sources is $1.91 \mathrm{~km} \mathrm{~s}^{-1}$, which is significantly larger than that $\left(0.99 \mathrm{~km} \mathrm{~s}^{-1}\right)$ of the non-outflow sources, indicating that outflow activities have a great influence on the strongly shocked gas. In particular, $\mathrm{SiO}$ emission clumps with small velocity dispersion could be formed by low-velocity shocks that are induced by either Hir regions or other large-scale compression flows (e.g., cloud-cloud collision), which will be investigated thoroughly in forthcoming works with higher resolution ALMA 12-m array data.

(3) We find a positive correlation between the $\mathrm{SiO}$ line luminosity and the bolometric luminosity, implying stronger shock activities associated with more luminous proto-clusters. We also find the $\mathrm{SiO}$ clumps with $\mathrm{H}_{\text {II }}$ regions show a lower $L_{\text {sio }} / L_{\text {bol }}$ ratio than the $\mathrm{SiO}$ clumps without $\mathrm{H}_{\mathrm{II}}$ regions. In most sources, the velocity dispersion of $\mathrm{SiO}$ emission and $[\mathrm{SiO}] /\left[\mathrm{H}^{13} \mathrm{CO}^{+}\right]$show no obvious correlations with dust temperature $\left(T_{\mathrm{d}}\right)$ and particle number density $(n)$. These results indicated that the $\mathrm{SiO}$ emission is not likely affected thermal conditions but is more likely affected by shock activities. In addition, we do not see clear correlations between the SiO line luminosity and $L_{\mathrm{bol}} / M$. There is also no robust trend in the $[\mathrm{SiO}] /\left[\mathrm{H}^{13} \mathrm{CO}^{+}\right]$ and $L_{\mathrm{bol}} / M$ relation. This implies that the fraction of shocked gas in dense gas does not change obviously at various evolutionary stages.

\section{ACKNOWLEDGEMENTS}

Tie Liu acknowledges the supports by National Natural Science Foundation of China (NSFC) through grants No.12073061 and No.12122307, the international partnership program of Chinese academy of sciences through grant No.114231KYSB20200009, and Shanghai Pujiang Program 20PJ1415500. This work is supported by the Ministry of Science and Technology of China through grant 2010DFA02710, the Key Project of Interntional Cooperation, and by the National Natural Science Foundation of China (NSFC) through grants 11503035,11573036 . H.-L. Liu is supported by NSFC through the grant No.12103045. K. W. acknowledges support by the National Science Foundation of China $(12041305,11973013)$ and the HighPerformance Computing Platform of Peking University through the instrumental analysis fund of Peking University (0000057511). This research was carried out in part at the Jet Propulsion Laboratory, which is operated by the California Institute of Technology under a contract with the National Aeronautics and Space Administration (80NM0018D0004). G.G. and L.B. acknowledge support by the ANID BASAL project FB210003. S.-L. Qin is supported by NSFC under grant No. 12033005. C. W. L. is supported by the Basic Science Research Program through the National Research Foundation of Korea (NRF) funded by the Ministry of Education, Science and Technology (NRF-2019R1A2C1010851). Y. Zhang acknowledges financial support from NSFC (grant No. 11973099). JHH thanks the National Natural Science Foundation of China under grant No. 11873086. This work is sponsored (in part) by the Chinese Academy of Sciences (CAS), through a grant to the CAS South America Center for Astronomy (CASSACA) in Santiago, Chile. Guo-Yin Zhang acknowledges support by China Postdoctoral Science Foundation (No. 2021T140672). This paper makes use of the following ALMA data: ADS/JAO.ALMA\#2019.1.00685.S. ALMA is a partnership of ESO (representing its member states), NSF (USA), and NINS (Japan), together with NRC (Canada), MOST and ASIAA (Taiwan), and KASI (Republic of Korea), in cooperation with the Republic of Chile.

\section{DATA AVAILABILITY}

The data underlying this article are available in the article and in ALMA archive.

\section{REFERENCES}

Bally J., 2016, ARA\&A, 54, 491

Bronfman L., Nyman L. A., May J., 1996, A\&AS, 115, 81

Churchwell E., 2002, in Hot Star Workshop III: The Earliest Phases of Massive Star Birth. p. 3

Codella C., Bachiller R., Reipurth B., 1999, A\&A, 343, 585

Cosentino G., et al., 2020, MNRAS, 499, 1666

Csengeri T., et al., 2016, A\&A, 586, A149

Duarte-Cabral A., Bontemps S., Motte F., Gusdorf A., Csengeri T., Schneider N., Louvet F., 2014, A\&A, 570, A1

Elia D., et al., 2021, MNRAS, 504, 2742

Gerner T., Beuther H., Semenov D., Linz H., Vasyunina T., Bihr S., Shirley Y. L., Henning T., 2014, A\&A, 563, A97

Gusdorf A., Cabrit S., Flower D. R., Pineau Des Forêts G., 2008a, A\&A, 482, 809

Gusdorf A., Pineau Des Forêts G., Cabrit S., Flower D. R., 2008b, A\&A, 490,695

Jiménez-Serra I., Caselli P., Tan J. C., Hernandez A. K., Fontani F., Butler M. J., van Loo S., 2010, MNRAS, 406, 187

Lefloch B., Castets A., Cernicharo J., Loinard L., 1998, ApJ, 504, L109

Leurini S., Codella C., López-Sepulcre A., Gusdorf A., Csengeri T., Anderl S., 2014, A\&A, 570, A49

Li S., et al., 2019a, ApJ, 878, 29

Li S., Zhang Q., Pillai T., Stephens I. W., Wang J., Li F., 2019b, ApJ, 886, 130

Li S., et al., 2020, ApJ, 903, 119

Liu T., et al., 2020a, MNRAS, 496, 2790

Liu T., et al., 2020b, MNRAS, 496, 2821

Liu H.-L., Sanhueza P., Liu T., Zavagno A., Tang X.-D., Wu Y., Zhang S., 2020c, ApJ, 901, 31

Liu H.-L., et al., 2021a, arXiv e-prints, p. arXiv:2111.02231

Liu H.-L., et al., 2021b, MNRAS, 505, 0035

Liu M., Tan J. C., Marvil J., Kong S., Rosero V., Caselli P., Cosentino G., 2021c, ApJ, 921, 96

López-Sepulcre A., et al., 2011, A\&A, 526, L2

López-Sepulcre A., Watanabe Y., Sakai N., Furuya R., Saruwatari O., Yamamoto S., 2016, ApJ, 822, 85 
Louvet F., et al., 2016, A\&A, 595, A122

Martin-Pintado J., Bachiller R., Fuente A., 1992, A\&A, 254, 315

McMullin J. P., Waters B., Schiebel D., Young W., Golap K., 2007, in Shaw R. A., Hill F., Bell D. J., eds, Astronomical Society of the Pacific Conference Series Vol. 376, Astronomical Data Analysis Software and Systems XVI. p. 127

Miettinen O., 2014, A\&A, 562, A3

Miettinen O., Harju J., Haikala L. K., Pomrén C., 2006, A\&A, 460, 721

Molinari S., Pezzuto S., Cesaroni R., Brand J., Faustini F., Testi L., 2008, A\&A, 481, 345

Molinari S., Merello M., Elia D., Cesaroni R., Testi L., Robitaille T., 2016, ApJ, 826, L8

Motte F., Bontemps S., Schilke P., Schneider N., Menten K. M., Broguière D., 2007, A\&A, 476, 1243

Motte F., Bontemps S., Louvet F., 2018, ARA\&A, 56, 41

Myers P. C., Mardones D., Tafalla M., Williams J. P., Wilner D. J., 1996, ApJ, 465, L133

Nomura H., Millar T. J., 2004, A\&A, 414, 409

Qiu K., Zhang Q., Beuther H., Yang J., 2007, ApJ, 654, 361

Sakai T., Sakai N., Hirota T., Yamamoto S., 2010, ApJ, 714, 1658

Sánchez-Monge Á., López-Sepulcre A., Cesaroni R., Walmsley C. M., Codella C., Beltrán M. T., Pestalozzi M., Molinari S., 2013, A\&A, 557, A94

Sanhueza P., Jackson J. M., Foster J. B., Garay G., Silva A., Finn S. C., 2012, ApJ, 756, 60

Schilke P., Walmsley C. M., Pineau des Forets G., Flower D. R., 1997, A\&A, 321, 293

Zinnecker H., Yorke H. W., 2007, ARA\&A, 45, 481

\section{REFERENCES}

Bally J., 2016, ARA\&A, 54, 491

Bronfman L., Nyman L. A., May J., 1996, A\&AS, 115, 81

Churchwell E., 2002, in Hot Star Workshop III: The Earliest Phases of Massive Star Birth. p. 3

Codella C., Bachiller R., Reipurth B., 1999, A\&A, 343, 585

Cosentino G., et al., 2020, MNRAS, 499, 1666

Csengeri T., et al., 2016, A\&A, 586, A149

Duarte-Cabral A., Bontemps S., Motte F., Gusdorf A., Csengeri T., Schneider N., Louvet F., 2014, A\&A, 570, A1

Elia D., et al., 2021, MNRAS, 504, 2742

Gerner T., Beuther H., Semenov D., Linz H., Vasyunina T., Bihr S., Shirley Y. L., Henning T., 2014, A\&A, 563, A97

Gusdorf A., Cabrit S., Flower D. R., Pineau Des Forêts G., 2008a, A\&A, 482, 809

Gusdorf A., Pineau Des Forêts G., Cabrit S., Flower D. R., 2008b, A\&A, 490, 695

Jiménez-Serra I., Caselli P., Tan J. C., Hernandez A. K., Fontani F., Butler M. J., van Loo S., 2010, MNRAS, 406, 187

Lefloch B., Castets A., Cernicharo J., Loinard L., 1998, ApJ, 504, L109

Leurini S., Codella C., López-Sepulcre A., Gusdorf A., Csengeri T., Anderl S., 2014, A\&A, 570, A49

Li S., et al., 2019a, ApJ, 878, 29

Li S., Zhang Q., Pillai T., Stephens I. W., Wang J., Li F., 2019b, ApJ, 886, 130

Li S., et al., 2020, ApJ, 903, 119

Liu T., et al., 2020a, MNRAS, 496, 2790

Liu T., et al., 2020b, MNRAS, 496, 2821

Liu H.-L., Sanhueza P., Liu T., Zavagno A., Tang X.-D., Wu Y., Zhang S., 2020c, ApJ, 901, 31

Liu H.-L., et al., 2021a, arXiv e-prints, p. arXiv:2111.02231

Liu H.-L., et al., 2021b, MNRAS, 505, 0035

Liu M., Tan J. C., Marvil J., Kong S., Rosero V., Caselli P., Cosentino G., 2021c, ApJ, 921, 96

López-Sepulcre A., et al., 2011, A\&A, 526, L2

López-Sepulcre A., Watanabe Y., Sakai N., Furuya R., Saruwatari O., Yamamoto S., 2016, ApJ, 822, 85

Louvet F., et al., 2016, A\&A, 595, A122
Martin-Pintado J., Bachiller R., Fuente A., 1992, A\&A, 254, 315

McMullin J. P., Waters B., Schiebel D., Young W., Golap K., 2007, in Shaw R. A., Hill F., Bell D. J., eds, Astronomical Society of the Pacific Conference Series Vol. 376, Astronomical Data Analysis Software and Systems XVI. p. 127

Miettinen O., 2014, A\&A, 562, A3

Miettinen O., Harju J., Haikala L. K., Pomrén C., 2006, A\&A, 460, 721

Molinari S., Pezzuto S., Cesaroni R., Brand J., Faustini F., Testi L., 2008, A\&A, 481, 345

Molinari S., Merello M., Elia D., Cesaroni R., Testi L., Robitaille T., 2016, ApJ, 826, L8

Motte F., Bontemps S., Schilke P., Schneider N., Menten K. M., Broguière D., 2007, A\&A, 476, 1243

Motte F., Bontemps S., Louvet F., 2018, ARA\&A, 56, 41

Myers P. C., Mardones D., Tafalla M., Williams J. P., Wilner D. J., 1996, ApJ, 465, L133

Nomura H., Millar T. J., 2004, A\&A, 414, 409

Qiu K., Zhang Q., Beuther H., Yang J., 2007, ApJ, 654, 361

Sakai T., Sakai N., Hirota T., Yamamoto S., 2010, ApJ, 714, 1658

Sánchez-Monge Á., López-Sepulcre A., Cesaroni R., Walmsley C. M., Codella C., Beltrán M. T., Pestalozzi M., Molinari S., 2013, A\&A, 557, A94

Sanhueza P., Jackson J. M., Foster J. B., Garay G., Silva A., Finn S. C., 2012, ApJ, 756, 60

Schilke P., Walmsley C. M., Pineau des Forets G., Flower D. R., 1997, A\&A, 321,293

Zinnecker H., Yorke H. W., 2007, ARA\&A, 45, 481

\section{APPENDIX A:}

Figure A1 present the moment maps and spectra for some exemplar sources. The images for all sources are available as on-line supplementary material. Tables A1, A2, and A3 show the derived parameters for $\mathrm{SiO}$ clumps.

This paper has been typeset

from a $\mathrm{T}_{\mathrm{E}} \mathrm{X} / \mathrm{L} \mathrm{T}_{\mathrm{E}} \mathrm{X}$ file prepared by the author. 

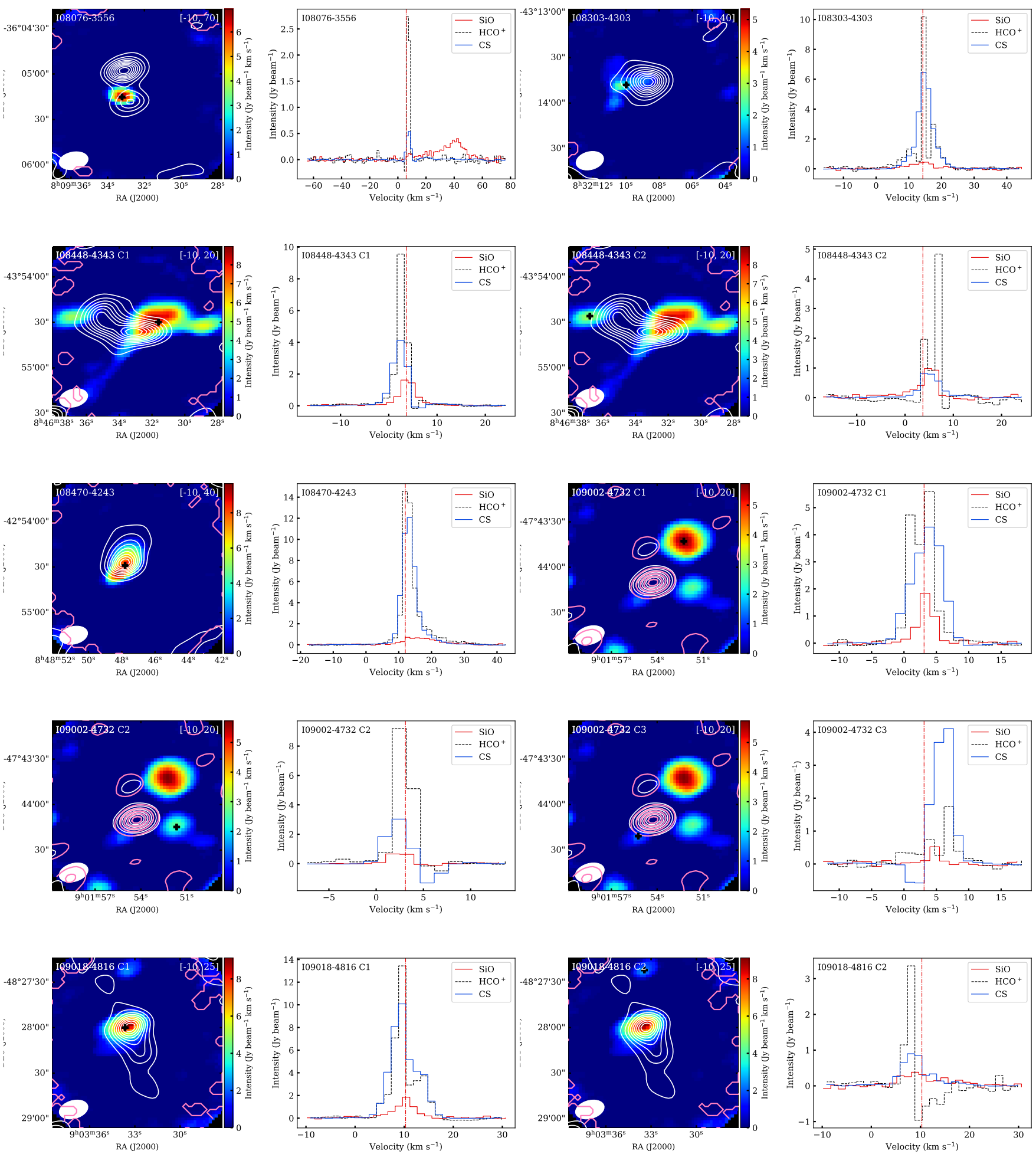

Figure A1. Examples of sources. Left: The background is $\mathrm{SiO}(2-1)$ integrated intensity maps. The white contours are $3 \mathrm{~mm}$ continuum emission, and contours are from $10 \%$ to $100 \%$ in the step of $10 \%$ of peak values. The pink contours represent $\mathrm{H} 40 \alpha$ emission, and contours are from $10 \%$ to $100 \%$ in the step of $20 \%$ of peak values. The black cross is the position extracted spectra. The source name is shown on the upper left. The integration velocity ranges are shown in the upper right corner. Right: The clump averaged $\mathrm{SiO}, \mathrm{HCO}^{+}$and $\mathrm{CS}$ spectra are shown in red, black dashed and blue lines, respectively. The vertical dash-dot line indicates the systemic velocity of the source. The full images are available as supplementary material. 
Table A1. SiO (2-1) Line Parameters and Clump Properties in the Region with Clear Detections

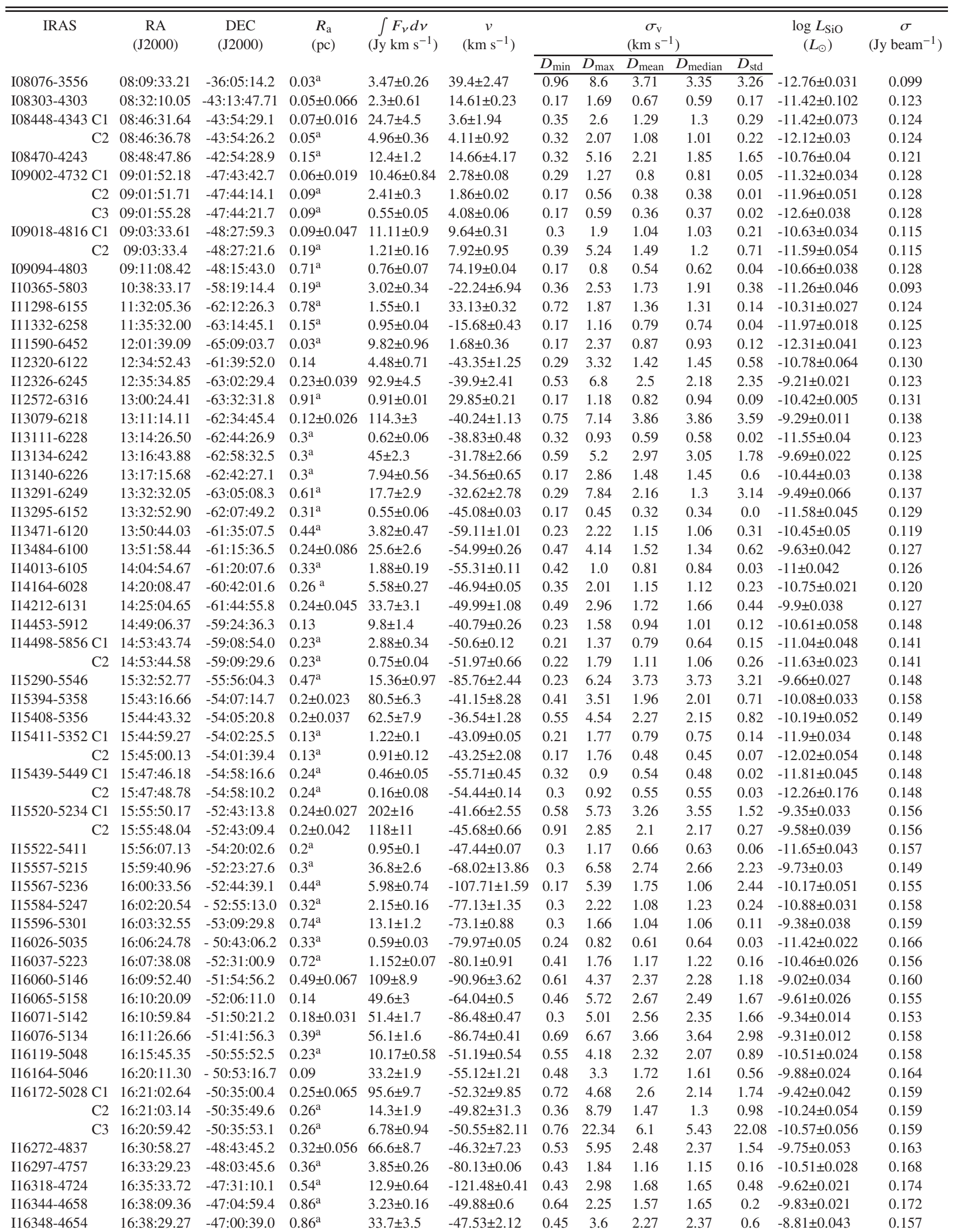




\begin{tabular}{|c|c|c|c|c|c|c|c|c|c|c|c|c|}
\hline \multirow[t]{2}{*}{ IRAS } & \multirow[t]{2}{*}{$\begin{array}{c}\mathrm{RA} \\
(\mathrm{J} 2000)\end{array}$} & \multirow[t]{2}{*}{$\begin{array}{c}\text { DEC } \\
(\mathrm{J} 2000)\end{array}$} & \multirow[t]{2}{*}{$\begin{array}{c}R_{\mathrm{a}} \\
(\mathrm{pc})\end{array}$} & \multirow[t]{2}{*}{$\begin{array}{c}\int F_{v} d v \\
\left(\mathrm{Jy}_{\mathrm{km} \mathrm{s}}{ }^{-1}\right)\end{array}$} & \multirow[t]{2}{*}{$\begin{array}{c}v \\
\left(\mathrm{~km} \mathrm{~s}^{-1}\right)\end{array}$} & \multicolumn{5}{|c|}{$\begin{array}{c}\sigma_{\mathrm{v}} \\
\left(\mathrm{km} \mathrm{s}^{-1}\right)\end{array}$} & \multirow[t]{2}{*}{$\begin{array}{c}\log L_{\mathrm{SiO}} \\
\left(L_{\odot}\right)\end{array}$} & \multirow[t]{2}{*}{$\begin{array}{c}\sigma \\
\left(\mathrm{Jy} \mathrm{beam}^{-1}\right)\end{array}$} \\
\hline & & & & & & $D_{\min }$ & $D_{\max }$ & $D_{\text {mean }}$ & $D_{\text {median }}$ & $D_{\text {std }}$ & & \\
\hline I16351-4722 & $16: 38: 50.40$ & $-47: 27: 58.3$ & $0.18 \pm 0.069$ & $79.4 \pm 8$ & $-40.4 \pm 4.29$ & 0.23 & 6.61 & 2.42 & 2.1 & 2.46 & $-9.64 \pm 0.042$ & 0.177 \\
\hline I16385-4619 & $16: 42: 13.74$ & $-46: 25: 29.8$ & $0.51^{\mathrm{a}}$ & $9.84 \pm 0.59$ & $-119.57 \pm 0.64$ & 0.53 & 2.96 & 1.76 & 1.66 & 0.49 & $-9.81 \pm 0.025$ & 0.176 \\
\hline I16424-4531 & $16: 46: 06.26$ & $-45: 36: 39.0$ & $0.18^{\mathrm{a}}$ & $1.78 \pm 0.14$ & $-31.75 \pm 30.16$ & 0.17 & 6.37 & 2.6 & 1.37 & 5.17 & $-11.41 \pm 0.033$ & 0.173 \\
\hline I16445-4459 & $16: 48: 04.54$ & $-45: 05: 03.9$ & $0.55^{\mathrm{a}}$ & $1.3 \pm 0.08$ & $-121.08 \pm 0.23$ & 0.24 & 1.09 & 0.8 & 0.85 & 0.05 & $-10.59 \pm 0.026$ & 0.164 \\
\hline I16458-4512 C1 & $16: 49: 29.22$ & $-45: 18: 09.3$ & $0.25^{\mathrm{a}}$ & $11.4 \pm 1.8$ & $-50.77 \pm 1.97$ & 0.36 & 4.93 & 1.63 & 1.53 & 0.89 & $-10.34 \pm 0.064$ & 0.167 \\
\hline $\mathrm{C} 2$ & $16: 49: 32.31$ & $-45: 17: 30.0$ & $0.25^{\mathrm{a}}$ & $0.78 \pm 0.08$ & $-49.19 \pm 0.15$ & 0.24 & 1.88 & 0.56 & 0.53 & 0.07 & & 0.167 \\
\hline I16484-4603 & $16: 52: 02.10$ & $-46: 08: 16.6$ & $0.15^{\mathrm{a}}$ & $17.1 \pm 1.5$ & $-33.15 \pm 1.62$ & 0.65 & 3.53 & 1.57 & 1.4 & 0.48 & $-10.63 \pm 0.037$ & 0.165 \\
\hline I16487-4423 & $16: 52: 23.92$ & $-44: 27: 47.9$ & $0.23^{\mathrm{a}}$ & $3.36 \pm 0.3$ & $-42.69 \pm 0.23$ & 0.17 & 1.22 & 0.74 & 0.84 & 0.1 & $-10.95 \pm 0.037$ & 0.169 \\
\hline I16489-4431 & $16: 52: 34.13$ & $-44: 36: 26.0$ & $0.22^{\mathrm{a}}$ & $6.98 \pm 0.6$ & $-40.45 \pm 1.61$ & 0.3 & 2.67 & 1.69 & 1.77 & 0.35 & $-10.63 \pm 0.036$ & 0.162 \\
\hline I16524-4300 C1 & $16: 56: 03.80$ & $-43: 04: 59.7$ & $0.24 \pm 0.083$ & $12.4 \pm 2.2$ & $-41.45 \pm 1.8$ & 0.35 & 2.17 & 1.24 & 1.36 & 0.22 & $-10.34 \pm 0.071$ & 0.106 \\
\hline $\mathrm{C} 2$ & $16: 56: 02.36$ & $-43: 04: 18.9$ & $0.15 \pm 0.089$ & $7.7 \pm 1.2$ & $-40.73 \pm 2.38$ & 0.17 & 2.33 & 1.09 & 0.95 & 0.38 & $-10.55 \pm 0.063$ & 0.106 \\
\hline $\mathrm{C} 3$ & $16: 56: 06.55$ & $-43: 04: 12.5$ & $0.25^{\mathrm{a}}$ & $0.81 \pm 0.1$ & $-42.1 \pm 0.2$ & 0.23 & 1.09 & 0.63 & 0.62 & 0.05 & $-11.52 \pm 0.051$ & 0.106 \\
\hline I16547-4247 C1 & $16: 58: 16.79$ & $-42: 52: 03.0$ & $0.17 \pm 0.057$ & $50.7 \pm 5.2$ & $-29.37 \pm 1.77$ & 0.51 & 4.58 & 2.26 & 2.32 & 1.25 & $-9.92 \pm 0.042$ & 0.119 \\
\hline $\mathrm{C} 2$ & $16: 58: 18.11$ & $-42: 52: 37.2$ & $0.2^{\mathrm{a}}$ & $3.81 \pm 0.92$ & $-36.64 \pm 2.34$ & 0.57 & 5.02 & 2.12 & 1.75 & 0.95 & $-11.05 \pm 0.094$ & 0.119 \\
\hline I16562-3959 C1 & $16: 59: 41.33$ & $-40: 03: 37.6$ & 0.14 & $36.8 \pm 3.9$ & $-11.74 \pm$ & 0.46 & 4.95 & 2.36 & 2.3 & 1.65 & 0.044 & 0.115 \\
\hline $\mathrm{C} 2$ & $16: 59: 38.93$ & $-40: 04: 11.0$ & $0.1 \pm 0.033$ & $20.9 \pm 1.6$ & $-11.4 \pm 0$ & 0.3 & 1.53 & 1.01 & 1.05 & 0.11 & 0.032 & 0.115 \\
\hline $\mathrm{C} 3$ & $16: 59: 43.59$ & $-40: 03: 08.5$ & 0.16 & $22.2 \pm 3$ & $-10.53 \pm 2.6$ & 0.37 & 2.55 & 1.44 & 1.54 & 0.26 & $-10.4 \pm 0.055$ & 0.115 \\
\hline I16571-4029 & $17: 00: 32.34$ & $-40: 34: 08.0$ & $0.17^{\mathrm{a}}$ & $15.2 \pm 0.68$ & $-13.78 \pm 1.32$ & 0.48 & 4.71 & 2.55 & 2.44 & 1.22 & $-10.57 \pm 0.019$ & 0.112 \\
\hline I17006-4215 & $17: 04: 11.88$ & $-42: 19: 50.0$ & $0.16^{\mathrm{a}}$ & $2.39 \pm 0.22$ & $-21.78 \pm 0.2$ & 0.23 & 2.23 & 1.21 & 1.19 & 0.35 & $-11.44 \pm 0.038$ & 0.117 \\
\hline I17008-4040 C1 & 17:04:22.96 & $-40: 44: 19.6$ & $0.16 \pm 0$ & $15.6 \pm 2.8$ & $-18.15 \pm 2.77$ & 0.23 & 2.54 & 1.35 & 1.36 & 0.4 & $-10.56 \pm 0.072$ & 0.120 \\
\hline $\mathrm{C} 2$ & $17: 04: 25.22$ & $-40: 44: 28.4$ & $0.17^{\mathrm{a}}$ & $5.44 \pm 0.74$ & -1 & 0.68 & 2.82 & 1.72 & 1.72 & 0.35 & -11 & 0.120 \\
\hline I17016-4124 C1 & $17: 05: 10.61$ & $-41: 29: 18.9$ & $0.14 \pm 0.037$ & $122 \pm 18$ & $-26.25 \pm 33.11$ & 0.65 & 6.52 & 3.5 & 3.25 & 2.81 & .06 & 0.116 \\
\hline $\mathrm{C} 2$ & $17: 05: 11.25$ & $-41: 28: 52.7$ & 0.09 & $96.1 \pm 9.2$ & $-31.02 \pm 14.83$ & 0.63 & 7.13 & 3.97 & 3.81 & 3.41 & -10.2 & 0.116 \\
\hline I17143-3700 & $17: 17: 45.50$ & $-37: 03: 12.5$ & $0.87^{\mathrm{a}}$ & $1.58 \pm 0.13$ & $-31.18 \pm 0.24$ & 0.36 & 1.38 & 0.87 & 0.92 & 0.04 & $-10.1 \pm 0.034$ & 0.140 \\
\hline I17158-3901 & $17: 19: 20.41$ & $-39: 03: 49.5$ & $0.12 \pm 0$ & $19.7 \pm 2.1$ & $-17.54 \pm 2.0$ & 0.34 & 3.11 & 1.65 & 1.59 & 0.6 & -10 & 0.159 \\
\hline I17160-3707 C1 & $17: 19: 26.61$ & $-37: 10: 21.4$ & 0.66 & $14 \pm 1.9$ & $-69.0 \pm$ & 0.42 & 2.89 & 1.69 & & 0.42 & $-9.31 \pm 0$ & 51 \\
\hline $\mathrm{C} 2$ & $17: 19: 25.83$ & $-37: 10: 02.8$ & $0.72^{\mathrm{a}}$ & $6.9 \pm 1.4$ & $-69.92 \pm 26.24$ & 0.62 & 18.32 & 6.3 & & 25.68 & & 0.151 \\
\hline $\mathrm{C} 3$ & $17: 19: 26.60$ & $-37: 11: 12.6$ & $0.72^{\mathrm{a}}$ & $1.11 \pm 0.09$ & $-69.17 \pm 0.21$ & 0.35 & 1.38 & 0.88 & 0.87 & 0.13 & .034 & 0.151 \\
\hline I17175-3544 C1 & $17: 20: 52.71$ & $-35: 47: 04.9$ & $0.09^{\mathrm{a}}$ & $65.5 \pm 9.8$ & $-9.18 \pm 13.26$ & 0.35 & 9.7 & 3.8 & 3.01 & 9.74 & -10 & 0.155 \\
\hline $\mathrm{C} 2$ & $17: 20: 54.55$ & $-35: 46: 46.8$ & $0.09^{\mathrm{a}}$ & $7.7 \pm 2.1$ & $1.17 \pm 0.5$ & 0.91 & 4.44 & 2.93 & 3.14 & 1.17 & $-11.36 \pm 0.105$ & 0.155 \\
\hline $\mathrm{C} 3$ & $17: 20: 50.26$ & $-35: 46: 40.7$ & $0.09^{\mathrm{a}}$ & $6.48 \pm 0.42$ & $0.88 \pm 10.1$ & 0.84 & 6.37 & 3.71 & 3.62 & 1.58 & $-11.44 \pm 0.027$ & 0.155 \\
\hline I17204-3636 & $17: 23: 50.18$ & $-36: 38: 56.7$ & $0.23^{\mathrm{a}}$ & $3.21 \pm 0.29$ & $-19.79 \pm 0.68$ & 0.36 & 2.23 & 1.29 & 1.48 & 0.24 & $-10.95 \pm 0.038$ & 0.132 \\
\hline I17220-3609 & $17: 2$ & $-36: 1$ & 0.48 & $25.6 \pm$ & & & & 1.72 & & 0.74 & & 4 \\
\hline I17233-3606 C1 & $17: 26: 40.91$ & $-36: 09: 26.8$ & $0.14 \pm 0$ & $13.8 \pm 9.6$ & 1.6 & 0.31 & 8.77 & 4.75 & 5.28 & 3.71 & -9 . & 0.169 \\
\hline $\mathrm{C} 2$ & $17: 26: 42.53$ & $-36: 09: 17.3$ & 0.07 & $159 \pm 19$ & $2.47 \pm$ & 0.83 & 10.5 & 4.77 & 4.35 & 7.03 & -10 & 0.169 \\
\hline $\mathrm{C} 3$ & $17: 26: 45.55$ & $-36: 09: 20.4$ & $0.15 \pm 0.031$ & $53.3 \pm 8.9$ & $0.99 \pm 0.51$ & 0.67 & 5.03 & 2.83 & 2.54 & 1.81 & $-10.52 \pm 0.067$ & 0.169 \\
\hline I17244-3536 & $17: 27: 50.28$ & $-35: 38: 57.8$ & $0.09^{\mathrm{a}}$ & $0.59 \pm 0.04$ & $-10.43 \pm 0.28$ & 0.46 & 1.44 & 1.15 & 1.15 & 0.04 & $-12.46 \pm 0.028$ & 0.148 \\
\hline I17258-3637 C1 & $17: 29: 18.35$ & $-36: 40: 27.8$ & $0.15 \pm 0.051$ & $12.1 \pm 1.6$ & $-13.37 \pm 2.06$ & 0.36 & 1.86 & 1.1 & 1.06 & 0.16 & $-10.59 \pm 0.054$ & 0.161 \\
\hline $\mathrm{C} 2$ & $17: 29: 18.65$ & $-36: 40: 50.0$ & $0.18^{\mathrm{a}}$ & $3.13 \pm 0.49$ & $-10.86 \pm 5.07$ & 0.26 & 1.45 & 0.89 & 0.85 & 0.09 & $-11.18 \pm 0.063$ & 0.161 \\
\hline I17269-3312 & $17: 30: 14.47$ & $-33: 15: 01.1$ & $0.3^{\mathrm{a}}$ & $10.73 \pm 0.69$ & $-24.11 \pm 2.5$ & 0.35 & 3.08 & 1.59 & 1.62 & 0.64 & \pm 0.027 & 0.149 \\
\hline I17271-3439 & $17: 30: 26.8$ & $-34: 41: 48.9$ & $0.37 \pm 0.091$ & $51.6 \pm 8$ & $4.81 \pm 0.0$ & 0.24 & 3.71 & 1.66 & 1.77 & 0.72 & $-9.81 \pm 0.063$ & 0.148 \\
\hline $\mathrm{I} 17278-3541 \mathrm{C} 1$ & $17: 31: 15.88$ & $-35: 44: 15.3$ & $0.09^{\mathrm{a}}$ & $6.45 \pm 0.46$ & $4.54 \pm 2.61$ & 0.37 & 3.29 & 1.97 & 2.22 & 0.84 & $-11.45 \pm 0.03$ & 0.151 \\
\hline $\mathrm{C} 2$ & $17: 31: 13.08$ & $-35: 43: 41.8$ & $0.09^{\mathrm{a}}$ & $3.55 \pm 0.17$ & $-8.39 \pm 4.01$ & 0.58 & 4.03 & 2.57 & 2.78 & 1.14 & $-11.7 \pm 0.02$ & 0.151 \\
\hline $\mathrm{C} 3$ & $17: 31: 16.02$ & $-35: 43: 27.2$ & $0.09^{\mathrm{a}}$ & $1.08 \pm 0.12$ & $0.94 \pm 0.25$ & 0.17 & 6.92 & 0.99 & 0.63 & 1.32 & $-12.22 \pm 0.046$ & 0.151 \\
\hline $\mathrm{C} 4$ & $17: 31: 16.52$ & $-35: 43: 46.0$ & $0.09^{\mathrm{a}}$ & $2.54 \pm 0.49$ & $9.51 \pm 27.86$ & 0.57 & 4.06 & 2.03 & 1.66 & 0.75 & $-11.85 \pm 0.077$ & 0.151 \\
\hline C5 & $17: 31: 13.26$ & $-35: 44: 07.9$ & $0.09^{\mathrm{a}}$ & $0.66 \pm 0$ & & 0.35 & 2.19 & 1.58 & 1.79 & 0.32 & & 0.151 \\
\hline C6 & $17: 31: 14.42$ & $-35: 43: 58.0$ & $0.09^{\mathrm{a}}$ & $0.43 \pm 0.05$ & $0.44 \pm 0.03$ & 0.17 & 0.81 & 0.51 & 0.61 & 0.05 & $-12.62 \pm 0.048$ & 0.151 \\
\hline I17439-2845 & $17: 47: 10.18$ & $-28: 45: 44.2$ & $0.53^{\mathrm{a}}$ & $0.44 \pm 0.01$ & $22.74 \pm 1.63$ & 0.74 & 3.6 & 2.29 & 2.63 & 0.73 & $-11.05 \pm 0.01$ & 0.105 \\
\hline I17441-2822 C1 & $17: 47: 22.78$ & $-28: 23: 15.1$ & $0.54^{\mathrm{a}}$ & $71 \pm 14$ & $60.87 \pm 22.29$ & 0.45 & 9.32 & 3.82 & 3.08 & 8.19 & $-8.83 \pm 0.078$ & 0.526 \\
\hline $\mathrm{C} 2$ & $17: 47: 18.57$ & $-28: 23: 15.8$ & $0.54^{\mathrm{a}}$ & $53 \pm 11$ & $58.9 \pm 50.44$ & 0.48 & 10.36 & 4.02 & 3.68 & 9.57 & $-8.96 \pm 0.082$ & 0.526 \\
\hline $\mathrm{C} 3$ & $17: 47: 22.06$ & $-28: 22: 49.4$ & $0.54^{\mathrm{a}}$ & $28.1 \pm 4.9$ & $64.93 \pm 40.02$ & 0.74 & 5.85 & 3.16 & 2.65 & 2.7 & $-9.24 \pm 0.07$ & 0.526 \\
\hline $\mathrm{C} 4$ & $17: 47: 18.30$ & $-28: 22: 46.6$ & $0.54^{\mathrm{a}}$ & $16.3 \pm 3.4$ & $59.47 \pm 14.94$ & 0.42 & 4.28 & 1.62 & 1.21 & 1.07 & $-9.47 \pm 0.082$ & 0.526 \\
\hline $\mathrm{C} 5$ & $17: 47: 20.82$ & $-28: 22: 59.5$ & $0.54^{\mathrm{a}}$ & $4.75 \pm 0.69$ & $45.77 \pm 0.4$ & 0.54 & 2.07 & 1.33 & 1.33 & 0.18 & $-10.01 \pm 0.059$ & 0.526 \\
\hline I17455-2800 & $17: 48: 41.59$ & $-28: 01: 56.2$ & $0.67^{\mathrm{a}}$ & $6.33 \pm 0.53$ & $-15.74 \pm 0.48$ & 0.24 & 2.02 & 1.11 & 1.07 & 0.27 & $-9.7 \pm 0.035$ & 0.129 \\
\hline I17545-2357 & $17: 57: 34.59$ & $-23: 57: 32.5$ & $0.2^{\mathrm{a}}$ & $1.43 \pm 0.38$ & $7.56 \pm 0.3$ & 0.17 & 1.28 & 0.42 & 0.3 & 0.05 & $-11.41 \pm 0.102$ & 0.100 \\
\hline I17589-2312 C1 & 18:01:59.12 & $-23: 12: 56.7$ & $0.21^{\mathrm{a}}$ & $3.83 \pm 0.65$ & $23.84 \pm 3.49$ & 0.32 & 1.96 & 1.03 & 0.92 & 0.15 & $-10.97 \pm 0.068$ & 0.098 \\
\hline $\mathrm{C} 2$ & 18:01:58.39 & $-23: 12: 21.1$ & $0.21^{\mathrm{a}}$ & $3.78 \pm 0.45$ & $19.88 \pm 1.68$ & 0.35 & 2.31 & 1.01 & 0.97 & 0.23 & $-10.98 \pm 0.049$ & 0.098 \\
\hline I18032-2032 & 18:06:15.05 & $-20: 31: 37.7$ & 0.18 & $64.8 \pm 3.1$ & & 0.38 & 10.76 & 4.26 & 3.77 & 8.97 & $-9.27 \pm 0.02$ & 0.088 \\
\hline I18056-1952 C1 & $18: 08: 38.16$ & $-19: 51: 50.1$ & 0.21 & $36.9 \pm 1.7$ & $66.88 \pm 0.82$ & 0.54 & 4.08 & 2.45 & 2.48 & 0.96 & $-9.07 \pm 0.02$ & 0.099 \\
\hline $\mathrm{C} 2$ & $18: 08: 38.00$ & $-19: 51: 12.5$ & $0.6^{\mathrm{a}}$ & $11.32 \pm 0.5$ & $65.47 \pm 5.17$ & 0.3 & 4.86 & 2.5 & 2.67 & 1.95 & $-9.59 \pm 0.021$ & 0.099 \\
\hline $\mathrm{C} 3$ & $18: 08: 36.37$ & $-19: 52: 14.3$ & $0.6^{\mathrm{a}}$ & $2.75 \pm 0.2$ & $70.98 \pm 4.31$ & 0.37 & 1.51 & 1.08 & 1.09 & 0.09 & $-10.2 \pm 0.03$ & 0.099 \\
\hline I18079-1756 C1 & $18: 10: 51.58$ & $-17: 55: 46.5$ & $0.13^{\mathrm{a}}$ & $3.31 \pm 0.43$ & $18.02 \pm 1.27$ & 0.17 & 1.09 & 0.63 & 0.67 & 0.08 & $-11.46 \pm 0.053$ & 0.139 \\
\hline $\mathrm{C} 2$ & $18: 10: 49.38$ & $-17: 56: 01.7$ & $0.13^{\mathrm{a}}$ & $1.37 \pm 0.1$ & $11.65 \pm 0.35$ & 0.29 & 1.43 & 0.93 & 1.01 & 0.06 & $-11.84 \pm 0.031$ & 0.139 \\
\hline I18089-1732 & $18: 11: 51.58$ & $-17: 31: 25.6$ & $0.18^{\mathrm{a}}$ & $5.83 \pm 0.28$ & $33.25 \pm 0.97$ & 0.3 & 2.74 & 1.54 & 1.46 & 0.43 & $-10.94 \pm 0.02$ & 0.136 \\
\hline
\end{tabular}




\begin{tabular}{|c|c|c|c|c|c|c|c|c|c|c|c|c|}
\hline \multirow[t]{2}{*}{ IRAS } & \multirow[t]{2}{*}{$\begin{array}{c}\text { RA } \\
(\mathrm{J} 2000)\end{array}$} & \multirow[t]{2}{*}{$\begin{array}{c}\text { DEC } \\
(\mathrm{J} 2000)\end{array}$} & \multirow[t]{2}{*}{$\begin{array}{l}R_{\mathrm{a}} \\
(\mathrm{pc})\end{array}$} & \multirow[t]{2}{*}{$\int_{(\mathrm{Jy} \mathrm{km} \mathrm{s}} F_{v} d v$} & \multirow[t]{2}{*}{$\begin{array}{c}v \\
\left(\mathrm{~km} \mathrm{~s}^{-1}\right)\end{array}$} & \multicolumn{5}{|c|}{$\begin{array}{c}\sigma_{\mathrm{v}} \\
\left(\mathrm{km} \mathrm{s}^{-1}\right)\end{array}$} & \multirow[t]{2}{*}{$\begin{array}{c}\log L_{\mathrm{SiO}} \\
\left(L_{\odot}\right)\end{array}$} & \multirow[t]{2}{*}{$\begin{array}{c}\sigma \\
\left(\mathrm{Jy} \mathrm{beam}^{-1}\right)\end{array}$} \\
\hline & & & & & & $D_{\min }$ & $D_{\max }$ & $D_{\text {mean }}$ & $D_{\text {median }}$ & $D_{\text {std }}$ & & \\
\hline I18117-1753 & $18: 14: 39.21$ & $-17: 52: 08.6$ & $0.21 \pm 0.066$ & $31.6 \pm 3.9$ & $35.6 \pm 9.08$ & 0.42 & 4.11 & 1.94 & 1.64 & 1.03 & $-10.18 \pm 0.051$ & 0.138 \\
\hline I18139-1842 & $18: 16: 51.47$ & $-18: 41: 39.5$ & $0.22^{\mathrm{a}}$ & $3.15 \pm 0.17$ & $39.36 \pm 0.27$ & 0.17 & 1.8 & 1.0 & 1.01 & 0.27 & $-11.04 \pm 0.023$ & 0.134 \\
\hline I18159-1648 & $18: 18: 54.84$ & $-16: 47: 50.4$ & 0.09 & $47.1 \pm 3.5$ & $20.13 \pm 4.86$ & 0.36 & 5.89 & 2.36 & 2.24 & 1.92 & $-10.49 \pm 0.031$ & 0.138 \\
\hline I18182-1433 & $18: 21: 09.03$ & $-14: 31: 46.0$ & $0.34^{\mathrm{a}}$ & $12.24 \pm 0.78$ & $59.76 \pm 0.63$ & 0.4 & 2.82 & 1.61 & 1.64 & 0.52 & $-10.07 \pm 0.027$ & 0.130 \\
\hline I18236-1205 & $18: 26: 26.50$ & $-12: 03: 53.4$ & $0.16^{\mathrm{a}}$ & $1.84 \pm 0.16$ & $27.13 \pm 6.9$ & 0.17 & 2.97 & 1.15 & 1.27 & 0.44 & $-11.57 \pm 0.036$ & 0.144 \\
\hline I18264-1152 & $18: 29: 14.91$ & $-11: 50: 25.6$ & $0.17 \pm 0.061$ & $19.4 \pm 2.7$ & $43.35 \pm 4.27$ & 0.55 & 4.88 & 1.78 & 1.35 & 1.04 & $-10.17 \pm 0.057$ & 0.137 \\
\hline I18290-0924 & $18: 31: 43.13$ & $-09: 22: 32.9$ & $0.39^{\mathrm{a}}$ & $1.87 \pm 0.24$ & $84.15 \pm 0.46$ & 0.3 & 1.38 & 0.81 & 0.8 & 0.08 & -10.78 & 0.101 \\
\hline I18311-0809 & $18: 33: 53.54$ & $-08: 07: 12.2$ & $0.45^{\mathrm{a}}$ & $3.35 \pm 0$ & $113.7 \pm 0.19$ & 0.32 & 2.3 & 1.44 & 1.47 & 0.33 & -10.4 & 0.096 \\
\hline I18316-0602 C1 & $18: 34: 21.17$ & $-06: 00: 14.5$ & $0.16^{\mathrm{a}}$ & $28.3 \pm 1.3$ & $35.61 \pm 12.71$ & 0.29 & 8.83 & 4.23 & 3.88 & 7.48 & -10.41 & 0.093 \\
\hline $\mathrm{C} 2$ & $18: 34: 20.52$ & $-05: 59: 33.6$ & $0.16^{\mathrm{a}}$ & $25.4 \pm 2.5$ & $43.5 \pm 33.06$ & 0.42 & 6.5 & 2.74 & 2.3 & 2.76 & -10.46 & 0.093 \\
\hline $\mathrm{C} 3$ & $18: 34: 20.52$ & $-05: 59: 33.6$ & $0.05 \pm 0$ & $6.6 \pm 2.6$ & $29.93 \pm 5.4$ & 1.05 & 7.78 & 2.48 & 1.91 & 2.37 & $-11.04 \pm$ & 0.093 \\
\hline I18341-0727 & $18: 36: 49.87$ & $-07: 24: 53.6$ & $0.45^{\mathrm{a}}$ & $9.6 \pm 1.1$ & $112.5 \pm 3.89$ & 0.29 & 2.97 & 1.52 & 1.45 & 0.52 & $-9.96 \pm 0.047$ & 0.096 \\
\hline I18411-0338 & $18: 43: 46.11$ & $-03: 35: 31.2$ & $0.57^{\mathrm{a}}$ & $5.23 \pm$ & $103.62 \pm 0.06$ & 0.23 & 1.82 & 1.08 & 1.18 & 0.22 & -10.0 & 0.115 \\
\hline I18434-0242 & $18: 46: 03.58$ & $-02: 39: 24.7$ & $0.39^{\mathrm{a}}$ & $19.81 \pm$ & $97.65 \pm 0.11$ & 0.42 & 4.24 & 2.19 & 2.06 & 1.29 & -9.7 & 0.094 \\
\hline I18461-0113 & $18: 48: 41.74$ & $-01: 10: 01.3$ & $0.39^{\mathrm{a}}$ & $14.65 \pm 0.59$ & $95.48 \pm 0.34$ & 0.46 & 2.53 & 1.58 & 1.63 & 0.35 & $-9.91 \pm 0.017$ & 0.094 \\
\hline I18469-0132 & $18: 49: 33.05$ & $-01: 29: 02.1$ & $0.39^{\mathrm{a}}$ & $4.57 \pm 0.24$ & $86.96 \pm 0.12$ & 0.17 & 1.72 & 1.04 & 0.92 & 0.18 & $-10.42 \pm 0.022$ & 0.123 \\
\hline I18479-0005 & $18: 50: 30.63$ & $-00: 01: 58.9$ & $0.99^{\mathrm{a}}$ & $8.42 \pm 0.57$ & $14.64 \pm 3.11$ & 0.45 & 2.84 & 1.83 & 1.85 & 0.46 & $-9.35 \pm 0.028$ & 0.097 \\
\hline I18507p0110 C1 & $18: 53: 18.50$ & $+01: 15: 01.2$ & $0.08 \pm 0$ & $35.1 \pm 6.1$ & $58.9 \pm 1.88$ & 0.32 & 5.8 & 2.0 & 1.95 & 1.8 & $-10.57 \pm 0.07$ & 0.126 \\
\hline $\mathrm{C} 2$ & $18: 53: 16.67$ & $+01: 15: 11.1$ & $0.08 \pm 0.029$ & $32.4 \pm 3.9$ & $61.56 \pm 5$ & 0.5 & 6.48 & 2.9 & 2.88 & 1.78 & $-10.61 \pm 0.049$ & 0.126 \\
\hline $\mathrm{C} 3$ & $18: 53: 15.62$ & $+01: 14: 20.1$ & $0.12^{\mathrm{a}}$ & $4.6 \pm 1.4$ & $54.97 \pm 2.24$ & 0.44 & 3.3 & 1.12 & 1.11 & 0.2 & $-11.45 \pm 0.115$ & 0.126 \\
\hline I18507p0121 C1 & $18: 53: 18.02$ & $+01: 25: 24.8$ & $0.12^{\mathrm{a}}$ & $48.7 \pm 4.8$ & $59.29 \pm 8.86$ & 0.23 & 4.78 & 2.29 & 2.19 & 2.27 & $-10.43 \pm 0.041$ & 0.110 \\
\hline $\mathrm{C} 2$ & $18: 53: 17.90$ & $+01: 24: 48.1$ & $0.09 \pm 0.03$ & $29 \pm 3.3$ & $57.25 \pm 7.38$ & 0.67 & 7.04 & 2.71 & 2.44 & 2.13 & $-10.65 \pm 0.047$ & 0.110 \\
\hline I18517p0437 & $18: 54: 14.50$ & $+04: 41: 39.3$ & $0.18^{\mathrm{a}}$ & $8 \pm 1$ & $43.85 \pm 0.23$ & 0.23 & 1.43 & 0.72 & 0.7 & 0.09 & $-10.85 \pm 0.051$ & 0.110 \\
\hline I18530p0215 & $18: 55: 33.42$ & $+02: 19: 01.6$ & $0.36^{\mathrm{a}}$ & $0.41 \pm 0.06$ & $77.43 \pm 3.21$ & 0.17 & 3.22 & 0.97 & 0.67 & 0.62 & $-11.55 \pm 0.059$ & 0.099 \\
\hline I19078p0901 & $19: 10: 13.24$ & $+09: 06: 14.0$ & $0.97 \pm 0.099$ & $278 \pm 17$ & $6.46 \pm 2.0$ & 0.88 & 6.91 & 3.81 & 4.0 & 2.27 & $-7.97 \pm 0.026$ & 0.122 \\
\hline I19095p0930 & $19: 11: 53.85$ & $+09: 35: 50.7$ & $0.43^{\mathrm{a}}$ & $18.7 \pm 1$ & $43.51 \pm 0.18$ & 0.95 & 3.32 & 2.16 & 2.22 & 0.4 & $-9.67 \pm 0.023$ & 0.116 \\
\hline I19097p0847 & 19:12:09.04 & $+08: 52: 12.2$ & $0.6^{\mathrm{a}}$ & $6.51 \pm 0.45$ & $57.27 \pm 0.38$ & 0.31 & 2.83 & 1.6 & 1.76 & 0.56 & $-9.83 \pm 0.029$ & 0.139 \\
\hline
\end{tabular}

Note. $R_{\mathrm{a}}$ : the linear radius of $\mathrm{SiO}$ clump. $\int F_{v} d v$ : the integrated intensity of $\mathrm{SiO}$ clump. $v$ : the velocity of $\mathrm{SiO}$ clump. $\sigma_{\mathrm{v}}$ : the velocity dispersion of $\mathrm{SiO}$ clump. $L_{\text {sio }}$ : the luminosity of the SiO clump. $\sigma:$ rms in one source.

a symbol after radius of $\mathrm{SiO}$ clump indicates that this $\mathrm{SiO}$ clump is unresolved by CASA.

Table A2. SiO (2-1) Line Parameters and Clump Properties in the Region Without Clear Detections

\begin{tabular}{|c|c|c|c|c|c|c|c|c|}
\hline IRAS & $\begin{array}{c}\text { RA } \\
\text { J2000 }\end{array}$ & $\begin{array}{c}\text { DEC } \\
\text { J2000 }\end{array}$ & $\begin{array}{l}R_{\mathrm{a}} \\
(\mathrm{pc})\end{array}$ & $\begin{array}{c}\int F_{v} d v \\
\left(\mathrm{Jy} \mathrm{km} \mathrm{s} \mathrm{kg}^{-1}\right)\end{array}$ & $\begin{array}{l}v \\
\left(\mathrm{~km} \mathrm{~s}^{-1}\right)\end{array}$ & $\begin{array}{c}\sigma_{\mathrm{v}} \\
\left(\mathrm{km} \mathrm{s}^{-1}\right)\end{array}$ & $\begin{array}{c}\log L_{\mathrm{SiO}} \\
\left(L_{\odot}\right)\end{array}$ & $\begin{array}{c}\sigma \\
\left(\text { Jy beam }^{-1}\right)\end{array}$ \\
\hline I12383-6128 & $12: 41: 18.18$ & $-61: 44: 17.4$ & $0.25^{\mathrm{a}}$ & $1.08 \pm 0.38$ & $-39 \pm 2$ & $1.3 \pm 1.7$ & $-11.44 \pm 0.13$ & 0.14 \\
\hline I13080-6229 & $13: 11: 12.81$ & $-62: 45: 02.1$ & $0.58 \pm 0.4$ & $3.6 \pm 1.4$ & $-35.7 \pm 8.1$ & $2.6 \pm 6.6$ & $-10.79 \pm 0.143$ & 0.122 \\
\hline I13291-6229 & $13: 32: 32.54$ & $-62: 45: 22.6$ & $0.35 \pm 0.18$ & $2.35 \pm 0.5$ & $-37.6 \pm 3.8$ & $0.99 \pm 3.1$ & $-11.21 \pm 0.08$ & 0.125 \\
\hline I14050-6056 & 14:08:40.89 & $-61: 11: 18.4$ & $0.28^{\mathrm{a}}$ & $3.81 \pm 0.99$ & $-53 \pm 3$ & $1.8 \pm 2.4$ & $-10.85 \pm 0.1$ & 0.154 \\
\hline I14382-6017 & 14:42:03.76 & $-60: 30: 30.9$ & $0.39 \pm 0.59$ & $3.6 \pm 1.7$ & $-60.7 \pm 2.6$ & $1.2 \pm 2.1$ & $-10.17 \pm 0.095$ & 0.153 \\
\hline I15254-5621 & $15: 29: 18.84$ & $-56: 31: 38.4$ & $0.28^{\mathrm{a}}$ & $4.1 \pm 1$ & $-68.5 \pm 2.3$ & $2.1 \pm 1.8$ & $-10.69 \pm 0.095$ & 0.16 \\
\hline I15437-5343 & $15: 47: 33.06$ & $-53: 52: 39.4$ & 0.46 & $4.9 \pm 1.4$ & $-83.1 \pm 2.8$ & $1.8 \pm 2.3$ & $-10.69 \pm 0.109$ & 0.163 \\
\hline I15502-5302 & $15: 54: 03.32$ & $-53: 11: 37.9$ & $0.43^{\mathrm{a}}$ & $3.91 \pm 0.74$ & $-92.4 \pm 1.7$ & $2 \pm 1.4$ & $-10.38 \pm 0.08$ & 0.176 \\
\hline I16304-4710 & $16: 34: 05.19$ & $-47: 16: 33.0$ & $0.8^{\mathrm{a}}$ & $1.42 \pm 0.48$ & $-62.3 \pm 2.3$ & $0.93 \pm 1.9$ & $-10.24 \pm 0.126$ & 0.211 \\
\hline I16313-4729 & $16: 34: 54.38$ & $-47: 35: 33.6$ & $0.33^{\mathrm{a}}$ & $5.4 \pm 1.2$ & $-74.3 \pm 2.2$ & $3 \pm 1.8$ & $-10.42 \pm 0.087$ & 0.188 \\
\hline I16330-4725 & $16: 36: 43.25$ & $-47: 31: 25.9$ & $0.78^{\mathrm{a}}$ & $4.1 \pm 1.2$ & $-71.4 \pm 2.7$ & $2.5 \pm 2.2$ & $-9.81 \pm 0.111$ & 0.205 \\
\hline I16372-4545 & $16: 40: 54.07$ & $-45: 50: 52.3$ & $0.3^{\mathrm{a}}$ & $2.8 \pm 0.5$ & $-58.2 \pm 2.4$ & $2 \pm 2$ & $-10.82 \pm 0.071$ & 0.204 \\
\hline I16506-4512 & $16: 54: 13.35$ & $-45: 17: 54.7$ & $0.07 \pm 0.05$ & $5.74 \pm 0.88$ & $-35.3 \pm 1.9$ & $3.8 \pm 1.6$ & $-10.98 \pm 0.062$ & 0.181 \\
\hline I17136-3617 & $17: 17: 02.31$ & $-36: 20: 49.4$ & $0.09^{\mathrm{a}}$ & $2.64 \pm 0.48$ & $-10.5 \pm 2.7$ & $2.8 \pm 2.2$ & $-11.83 \pm 0.073$ & 0.176 \\
\hline I18110-1854 & $18: 14: 02.41$ & $-18: 53: 14.1$ & $0.39 \pm 0.157$ & $13.5 \pm 4.1$ & $38.5 \pm 2.5$ & $3 \pm 2$ & $-10.32 \pm 0.115$ & 0.144 \\
\hline I18116-1646 & $18: 14: 36.17$ & $-16: 45: 45.7$ & $0.29^{\mathrm{a}}$ & $1.43 \pm 0.52$ & $48.6 \pm 2.5$ & $1.3 \pm 2$ & $-11.16 \pm 0.135$ & 0.145 \\
\hline I18223-1243 & $18: 25: 11.12$ & $-12: 42: 21.1$ & $0.24^{\mathrm{a}}$ & $1.31 \pm 0.33$ & $48.3 \pm 2.4$ & $2 \pm 2$ & $-10.95 \pm 0.076$ & 0.168 \\
\hline I18228-1312 & $18: 25: 41.66$ & $-13: 10: 11.6$ & 0.29 & $4.24 \pm 0.93$ & $31.7 \pm 2.7$ & $0.77 \pm 2.2$ & $-10.86 \pm 0.086$ & 0.148 \\
\hline
\end{tabular}

Note. $R_{\mathrm{a}}$ : The linear radius of $\mathrm{SiO}$ clump. $\int F_{v} d v$ : The integrated intensity of $\mathrm{SiO}$ clump. $v$ : SiO line central velocity. $\sigma_{\mathrm{v}}$ : The velocity dispersion of $\mathrm{SiO}$ clump. $L_{\mathrm{sio}}$ : The luminosity of the $\mathrm{SiO}$ clump. $\sigma: \mathrm{rms}$ in one source.

a symbol after radius of $\mathrm{SiO}$ clump indicates that this $\mathrm{SiO}$ clump is unresolved by CASA. 
Table A3. SiO (2-1) Line Parameters and Clump Properties

\begin{tabular}{|c|c|c|c|c|c|c|c|c|c|c|}
\hline \multirow[t]{3}{*}{ IRAS } & \multirow{3}{*}{$\begin{array}{c}L_{\mathrm{bol}} / M \\
\left(L_{\odot} M_{\odot}^{-1}\right)\end{array}$} & \multirow{3}{*}{$\begin{array}{c}\log n \\
\left(\mathrm{~cm}^{-3}\right)\end{array}$} & \multirow[t]{3}{*}[\mathrm{SiO}]{$/\left[\mathrm{H}^{13} \mathrm{CO}^{+}\right]$} & \multirow[t]{3}{*}{$D_{\mathrm{d}}$} & \multirow[t]{3}{*}{$\mathrm{H} 40 \alpha$} & \multicolumn{3}{|c|}{ Wing } & \multirow[t]{3}{*}{ outflow } & \multirow[t]{3}{*}{ type } \\
\hline & & & & & & $\mathrm{SiO}$ & $\mathrm{HCO}^{+}$ & $\mathrm{CS}$ & & \\
\hline & & & & & & & & & & \\
\hline I08076-3556 & 3.16 & 5.51 & - & 0.01 & & $\checkmark$ & & & $\checkmark$ & $\mathrm{C}$ \\
\hline I08303-4303 & 25.12 & 5.4 & $0.41 \pm 0.01$ & 0.16 & & $\checkmark$ & $\checkmark$ & $\checkmark$ & $\checkmark$ & $\mathrm{D}$ \\
\hline I08448-4343 C1 & 25.12 & 5.68 & $1.86 \pm 0.07$ & 0.06 & & $\checkmark$ & & & $\checkmark$ & $\mathrm{D}$ \\
\hline $\mathrm{C} 2$ & 25.12 & 5.68 & $3.35 \pm 0.37$ & 0.08 & & $\checkmark$ & & & $\checkmark$ & $\mathrm{D}$ \\
\hline I08470-4243 & 39.81 & 5.4 & $2.98 \pm 0.1$ & 0.02 & & $\checkmark$ & $\checkmark$ & $\checkmark$ & $\checkmark$ & $\mathrm{C}$ \\
\hline I09002-4732 C1 & 158.49 & 5.78 & $0.74 \pm-$ & 0.2 & $\checkmark$ & & & & & B \\
\hline $\mathrm{C} 2$ & 158.49 & 5.78 & - & 0.15 & $\checkmark$ & & & & & B \\
\hline $\mathrm{C} 3$ & 158.49 & 5.78 & - & 0.08 & $\checkmark$ & & & & & B \\
\hline I09018-4816 C1 & 50.12 & 5.59 & $0.9 \pm-$ & 0.05 & & & & & & $\mathrm{C}$ \\
\hline $\mathrm{C} 2$ & 50.12 & 5.59 & - & 0.48 & & & & & & $\mathrm{D}$ \\
\hline I09094-4803 & 31.62 & 4.18 & $1.39 \pm 0.16$ & 0.93 & & & & & & $\mathrm{D}$ \\
\hline I10365-5803 & 39.81 & 5.29 & $0.32 \pm-$ & 0.11 & & $\checkmark$ & $\checkmark$ & & $\checkmark$ & $\mathrm{D}$ \\
\hline I11298-6155 & 63.1 & 4.52 & $3.97 \pm 0.19$ & 0.37 & $\checkmark$ & & $\checkmark$ & $\checkmark$ & $\checkmark$ & $\mathrm{A}$ \\
\hline I11332-6258 & 39.81 & 5.28 & $0.22 \pm 0.05$ & 0.03 & & & $\checkmark$ & & $\checkmark$ & $\mathrm{C}$ \\
\hline I11590-6452 & 3.98 & 6.29 & - & 0.04 & & & & & & $\mathrm{D}$ \\
\hline I12320-6122 & 398.11 & 3.61 & $0.7 \pm 0.25$ & 0.25 & $\checkmark$ & $\checkmark$ & $\checkmark$ & $\checkmark$ & $\checkmark$ & B \\
\hline I12326-6245 & 79.43 & 4.36 & $6.16 \pm 0.12$ & 0.07 & $\checkmark$ & $\checkmark$ & $\checkmark$ & $\checkmark$ & $\checkmark$ & A \\
\hline I12383-6128 & 5.01 & 4.32 & $0.99 \pm 0.25$ & 0.37 & $\checkmark$ & & & & & B \\
\hline I12572-6316 & 5.01 & 3.88 & $0.42 \pm 0.05$ & 0.12 & $\checkmark$ & & & & & A \\
\hline I13079-6218 & 39.81 & 4 & $4.77 \pm 0.05$ & 0.11 & $\checkmark$ & $\checkmark$ & $\checkmark$ & $\checkmark$ & $\checkmark$ & A \\
\hline I13080-6229 & 79.43 & 3.78 & $0.15 \pm 0.01$ & 0.17 & $\checkmark$ & $\checkmark$ & & & $\checkmark$ & B \\
\hline I13111-6228 & 50.12 & 3.77 & $0.09 \pm-$ & 0.04 & $\checkmark$ & & $\checkmark$ & & $\checkmark$ & A \\
\hline I13134-6242 & 31.62 & 4.45 & $3.04 \pm 0.14$ & 0.08 & & $\checkmark$ & $\checkmark$ & $\checkmark$ & $\checkmark$ & $\mathrm{C}$ \\
\hline I13140-6226 & 7.94 & 4.42 & $1 \pm-$ & 0.05 & & $\checkmark$ & $\checkmark$ & $\checkmark$ & $\checkmark$ & $\mathrm{C}$ \\
\hline I13291-6229 & 39.81 & 4.09 & $0.13 \pm 0.01$ & 0.38 & $\checkmark$ & & & & & B \\
\hline I13291-6249 & 31.62 & 3.6 & $1.85 \pm 0.05$ & 0.5 & $\checkmark$ & $\checkmark$ & $\checkmark$ & $\checkmark$ & $\checkmark$ & B \\
\hline I13295-6152 & 3.98 & 4.64 & $0.09 \pm-$ & 0.14 & & & & & & $\mathrm{C}$ \\
\hline I13471-6120 & 79.43 & 4 & $0.62 \pm 0.09$ & 0.43 & $\checkmark$ & $\checkmark$ & $\checkmark$ & $\checkmark$ & $\checkmark$ & B \\
\hline I13484-6100 & 50.12 & 3.94 & $1.24 \pm 0.05$ & 0.14 & & $\checkmark$ & $\checkmark$ & $\checkmark$ & $\checkmark$ & $\mathrm{C}$ \\
\hline I14013-6105 & 63.1 & 4.1 & - & 0.08 & $\checkmark$ & & & & & A \\
\hline I14050-6056 & 79.43 & 3.95 & - & 0.61 & $\checkmark$ & & & & & B \\
\hline I14164-6028 & 31.62 & 5.12 & $0.94 \pm 0.04$ & 0.04 & & & & & & $\mathrm{C}$ \\
\hline I14212-6131 & 10.0 & 4.35 & $1.75 \pm 0.12$ & 0.36 & & $\checkmark$ & $\checkmark$ & $\checkmark$ & $\checkmark$ & $\mathrm{D}$ \\
\hline I14382-6017 & 39.81 & 3.54 & - & 0.58 & $\checkmark$ & & & & & B \\
\hline I14453-5912 & 19.95 & 3.82 & $1.65 \pm 0.45$ & 0.33 & $\checkmark$ & & & & & B \\
\hline I14498-5856 C1 & 25.12 & 4.01 & $0.52 \pm 0.01$ & 0.12 & & & $\checkmark$ & & $\checkmark$ & $\mathrm{D}$ \\
\hline $\mathrm{C} 2$ & 25.12 & 4.01 & - & 0.59 & & & & & & $\mathrm{D}$ \\
\hline I15254-5621 & 100.0 & 3.87 & - & 0.35 & $\checkmark$ & $\checkmark$ & & $\checkmark$ & $\checkmark$ & B \\
\hline I15290-5546 & 79.43 & 3.65 & $2.31 \pm 0.03$ & 0.18 & $\checkmark$ & $\checkmark$ & $\checkmark$ & $\checkmark$ & $\checkmark$ & A \\
\hline I15394-5358 & 6.31 & 4.53 & $1.75 \pm 0.03$ & 0.02 & & $\checkmark$ & $\checkmark$ & $\checkmark$ & $\checkmark$ & $\mathrm{C}$ \\
\hline I15408-5356 & 100.0 & 4.16 & $3.85 \pm 0.54$ & 0.26 & $\checkmark$ & $\checkmark$ & $\checkmark$ & $\checkmark$ & $\checkmark$ & B \\
\hline I15411-5352 C1 & 63.1 & 4.05 & $0.22 \pm-$ & 0.08 & $\checkmark$ & $\checkmark$ & & $\checkmark$ & $\checkmark$ & B \\
\hline $\mathrm{C} 2$ & 63.1 & 4.05 & $0.33 \pm-$ & 0.34 & $\checkmark$ & & & & & B \\
\hline I15437-5343 & 39.81 & 3.95 & $0.42 \pm 0.11$ & 0.11 & & & & & & $\mathrm{C}$ \\
\hline I15439-5449 C1 & 25.12 & 4.1 & $0.77 \pm 0.001$ & 0.57 & $\checkmark$ & & & & & B \\
\hline $\mathrm{C} 2$ & 25.12 & 4.1 & $0.04 \pm 0.003$ & 0.41 & $\checkmark$ & & & & & B \\
\hline I15502-5302 & 125.89 & 3.65 & $1.04 \pm 0.02$ & 0.81 & $\checkmark$ & & & $\checkmark$ & $\checkmark$ & B \\
\hline I15520-5234 C1 & 79.43 & 4.34 & $16.45 \pm 4.935$ & 0.18 & $\checkmark$ & $\checkmark$ & $\checkmark$ & $\checkmark$ & $\checkmark$ & B \\
\hline $\mathrm{C} 2$ & 79.43 & 4.34 & $4.06 \pm 0.005$ & 0.06 & $\checkmark$ & $\checkmark$ & & $\checkmark$ & $\checkmark$ & A \\
\hline I15522-5411 & 7.94 & 4.09 & $0.33 \pm 0.002$ & 0.04 & & $\checkmark$ & & $\checkmark$ & $\checkmark$ & $\mathrm{C}$ \\
\hline I15557-5215 & 5.01 & 4.07 & $4.81 \pm 0.144$ & 0.04 & & $\checkmark$ & $\checkmark$ & $\checkmark$ & $\checkmark$ & $\mathrm{C}$ \\
\hline I15567-5236 & 158.49 & 3.76 & $0.38 \pm 0.051$ & 0.29 & $\checkmark$ & $\checkmark$ & $\checkmark$ & $\checkmark$ & $\checkmark$ & B \\
\hline I15584-5247 & 12.59 & 3.91 & $0.62 \pm 0.043$ & 0.4 & & $\checkmark$ & $\checkmark$ & $\checkmark$ & $\checkmark$ & $\mathrm{D}$ \\
\hline I15596-5301 & 39.81 & 3.74 & $0.87 \pm 0.033$ & 0.34 & & & & & & $\mathrm{C}$ \\
\hline I16026-5035 & 79.43 & 3.89 & $0.36 \pm 0.07$ & 0.31 & & & & & & $\mathrm{D}$ \\
\hline I16037-5223 & 63.1 & 3.42 & $0.23 \pm 0.028$ & 0.1 & $\checkmark$ & $\checkmark$ & $\checkmark$ & $\checkmark$ & $\checkmark$ & A \\
\hline I16060-5146 & 79.43 & 4.23 & - & 0.08 & $\checkmark$ & $\checkmark$ & $\checkmark$ & $\checkmark$ & $\checkmark$ & A \\
\hline I16065-5158 & 50.12 & 3.87 & $1.5 \pm 0.061$ & 0.08 & $\checkmark$ & $\checkmark$ & $\checkmark$ & $\checkmark$ & $\checkmark$ & A \\
\hline I16071-5142 & 12.59 & 3.07 & $2.77 \pm 0.022$ & 0.08 & & $\checkmark$ & $\checkmark$ & $\checkmark$ & $\checkmark$ & $\mathrm{C}$ \\
\hline I16076-5134 & 50.12 & 3.63 & $5.61 \pm 0.016$ & 0 & & $\checkmark$ & $\checkmark$ & $\checkmark$ & $\checkmark$ & $\mathrm{C}$ \\
\hline I16119-5048 & 12.59 & 3.91 & $2.08 \pm 0.141$ & 0.07 & & $\checkmark$ & $\checkmark$ & $\checkmark$ & $\checkmark$ & $\mathrm{C}$ \\
\hline I16164-5046 & 63.1 & 3.9 & $0.92 \pm 0.091$ & 0.05 & $\checkmark$ & $\checkmark$ & & $\checkmark$ & $\checkmark$ & A \\
\hline
\end{tabular}




\begin{tabular}{|c|c|c|c|c|c|c|c|c|c|c|}
\hline \multirow[t]{3}{*}{ IRAS } & \multirow{3}{*}{$\begin{array}{c}L_{\mathrm{bol}} / M \\
\left(L_{\odot} M_{\odot}^{-1}\right)\end{array}$} & \multirow{3}{*}{$\begin{array}{c}\log n \\
\left(\mathrm{~cm}^{-3}\right)\end{array}$} & \multirow[t]{3}{*}[\mathrm{SiO}]{$/\left[\mathrm{H}^{13} \mathrm{CO}^{+}\right]$} & \multirow{3}{*}{$\begin{array}{l}D_{\mathrm{d}} \\
(\mathrm{pc})\end{array}$} & \multirow[t]{3}{*}{$\mathrm{H} 40 \alpha$} & \multicolumn{3}{|c|}{ Wing } & \multirow[t]{3}{*}{ outflow } & \multirow[t]{3}{*}{ type } \\
\hline & & & & & & $\mathrm{SiO}$ & $\mathrm{HCO}^{+}$ & $\mathrm{CS}$ & & \\
\hline & & & & & & & & & & \\
\hline I16172-5028 C1 & 63.1 & 4.08 & $1.27 \pm 0.03$ & 0.23 & $\checkmark$ & $\checkmark$ & $\checkmark$ & $\checkmark$ & $\checkmark$ & B \\
\hline $\mathrm{C} 2$ & 63.1 & 4.08 & - & 0.65 & $\checkmark$ & & & $\checkmark$ & $\checkmark$ & B \\
\hline $\mathrm{C} 3$ & 63.1 & 4.08 & - & 0.89 & $\checkmark$ & $\checkmark$ & & $\checkmark$ & $\checkmark$ & B \\
\hline I16272-4837 & 12.59 & 4.04 & $2.53 \pm 0.861$ & 0.14 & & $\checkmark$ & & $\checkmark$ & $\checkmark$ & $\mathrm{D}$ \\
\hline I16297-4757 & 31.62 & 3.11 & $0.73 \pm 0.055$ & 0.14 & $\checkmark$ & & & & & A \\
\hline I16304-4710 & 25.12 & 3.7 & $0.26 \pm 0.01$ & 0.22 & $\checkmark$ & & & & & A \\
\hline I16313-4729 & 100.0 & 4.37 & $0.7 \pm 0.04$ & 0.13 & & & & & & $\mathrm{C}$ \\
\hline I16318-4724 & 31.62 & 3.83 & $1.02 \pm 0.042$ & 0.09 & & $\checkmark$ & $\checkmark$ & $\checkmark$ & $\checkmark$ & $\mathrm{C}$ \\
\hline I16330-4725 & 100.0 & 3.14 & $0.23 \pm-$ & 0.19 & $\checkmark$ & & & & & A \\
\hline I16344-4658 & 19.95 & 3.42 & $0.63 \pm 0.028$ & 0.24 & & $\checkmark$ & & $\checkmark$ & $\checkmark$ & $\mathrm{C}$ \\
\hline I16348-4654 & 10.0 & 3.87 & $2.14 \pm 0.008$ & 0.3 & $\checkmark$ & $\checkmark$ & & $\checkmark$ & $\checkmark$ & A \\
\hline I16351-4722 & 50.12 & 4.3 & $2.27 \pm 0.114$ & 0.05 & & $\checkmark$ & & $\checkmark$ & $\checkmark$ & $\mathrm{C}$ \\
\hline I16372-4545 & 19.95 & 3.92 & $0.51 \pm 0.02$ & 0.2 & & & & & & $\mathrm{D}$ \\
\hline I16385-4619 & 79.43 & 3.43 & $1.51 \pm 0.126$ & 0.06 & $\checkmark$ & $\checkmark$ & $\checkmark$ & $\checkmark$ & $\checkmark$ & A \\
\hline I16424-4531 & 15.85 & 4.12 & $0.74 \pm 0.143$ & 0.12 & & $\checkmark$ & & $\checkmark$ & $\checkmark$ & $\mathrm{D}$ \\
\hline I16445-4459 & 12.59 & 3.3 & $0.33 \pm 0.07$ & 0.39 & & & & & & $\mathrm{D}$ \\
\hline I16458-4512 C1 & 7.94 & 3.76 & $4.39 \pm 0.054$ & 0.46 & $\checkmark$ & $\checkmark$ & & $\checkmark$ & $\checkmark$ & B \\
\hline $\mathrm{C} 2$ & 7.94 & 3.76 & - & 0.47 & $\checkmark$ & & & & & B \\
\hline I16484-4603 & 100.0 & 4.39 & $2.65 \pm 0.346$ & 0.33 & & $\checkmark$ & & & $\checkmark$ & $\mathrm{D}$ \\
\hline I16487-4423 & 25.12 & 3.6 & $1.31 \pm 0.071$ & 0.4 & & $\checkmark$ & & & $\checkmark$ & $\mathrm{D}$ \\
\hline I16489-4431 & 7.94 & 3.96 & $1.09 \pm 0.007$ & 0.24 & & $\checkmark$ & & $\checkmark$ & $\checkmark$ & $\mathrm{D}$ \\
\hline I16506-4512 & 79.43 & 3.91 & $3.58 \pm 0.67$ & 0.55 & $\checkmark$ & & & $\checkmark$ & $\checkmark$ & B \\
\hline I16524-4300 C1 & 10.0 & 3.94 & $1.48 \pm 0.13$ & 0.3 & & & & & & $\mathrm{D}$ \\
\hline $\mathrm{C} 2$ & 10.0 & 3.94 & $3.11 \pm 0.262$ & 0.47 & & & & & & $\mathrm{D}$ \\
\hline $\mathrm{C} 3$ & 10.0 & 3.94 & - & 0.87 & & & & & & $\mathrm{D}$ \\
\hline I16547-4247 C1 & 39.81 & 4.3 & $0.73 \pm 0.06$ & 0.08 & & $\checkmark$ & $\checkmark$ & $\checkmark$ & $\checkmark$ & $\mathrm{C}$ \\
\hline $\mathrm{C} 2$ & 39.81 & 4.3 & $16.68 \pm 7.892$ & 0.43 & & $\checkmark$ & $\checkmark$ & $\checkmark$ & $\checkmark$ & $\mathrm{D}$ \\
\hline I16562-3959 C1 & 316.23 & 4.24 & $0.8 \pm 0.197$ & 0.05 & & $\checkmark$ & $\checkmark$ & & $\checkmark$ & $\mathrm{C}$ \\
\hline $\mathrm{C} 2$ & 316.23 & 4.24 & - & 0.5 & & & & & & $\mathrm{D}$ \\
\hline $\mathrm{C} 3$ & 316.23 & 4.24 & - & 0.47 & & & & & & $\mathrm{D}$ \\
\hline I16571-4029 & 25.12 & 4.77 & $2.59 \pm 0.083$ & 0.02 & & $\checkmark$ & $\checkmark$ & $\checkmark$ & $\checkmark$ & $\mathrm{C}$ \\
\hline I17006-4215 & 39.81 & 4.32 & $0.61 \pm 0.087$ & 0.14 & $\checkmark$ & $\checkmark$ & & & $\checkmark$ & B \\
\hline I17008-4040 & 39.81 & 3.31 & $0.57 \pm 0.025$ & 0.05 & & $\checkmark$ & & & $\checkmark$ & $\mathrm{C}$ \\
\hline $\mathrm{C} 2$ & 39.81 & 3.31 & $42.78 \pm 31.127$ & 0.3 & & & & & & $\mathrm{D}$ \\
\hline I17016-4124 C1 & 31.62 & 4.79 & $6.61 \pm 0.19$ & 0.09 & & $\checkmark$ & $\checkmark$ & $\checkmark$ & $\checkmark$ & $\mathrm{D}$ \\
\hline $\mathrm{C} 2$ & 31.62 & 4.79 & $8.95 \pm 1.579$ & 0.08 & & $\checkmark$ & $\checkmark$ & $\checkmark$ & $\checkmark$ & $\mathrm{D}$ \\
\hline I17136-3617 & 125.89 & 4.15 & - & 0.14 & $\checkmark$ & $\checkmark$ & & $\checkmark$ & $\checkmark$ & B \\
\hline I17143-3700 & 63.1 & 3.0 & $0.19 \pm 0.007$ & 0.11 & $\checkmark$ & & & $\checkmark$ & $\checkmark$ & A \\
\hline I17158-3901 & 25.12 & 3.8 & $0.88 \pm 0.02$ & 0.04 & & $\checkmark$ & & & $\checkmark$ & $\mathrm{C}$ \\
\hline I17160-3707 C1 & 79.43 & 4.03 & $1.16 \pm 0.11$ & 2.15 & $\checkmark$ & $\checkmark$ & & & $\checkmark$ & $\mathrm{B}$ \\
\hline $\mathrm{C} 2$ & 79.43 & 4.03 & - & 1.4 & $\checkmark$ & & & $\checkmark$ & $\checkmark$ & B \\
\hline $\mathrm{C} 3$ & 79.43 & 4.03 & $0.21 \pm 0.024$ & 3.35 & $\checkmark$ & $\checkmark$ & & $\checkmark$ & $\checkmark$ & B \\
\hline I17175-3544 C1 & 50.12 & 5.08 & $1.9 \pm 0.014$ & 0.07 & $\checkmark$ & $\checkmark$ & $\checkmark$ & $\checkmark$ & $\checkmark$ & $\mathrm{B}$ \\
\hline $\mathrm{C} 2$ & 50.12 & 5.08 & - & 0.12 & $\checkmark$ & $\checkmark$ & & & $\checkmark$ & B \\
\hline $\mathrm{C} 3$ & 50.12 & 5.08 & $1.66 \pm 0.274$ & 0.29 & $\checkmark$ & $\checkmark$ & $\checkmark$ & $\checkmark$ & $\checkmark$ & B \\
\hline I17204-3636 & 19.95 & 4.18 & $0.28 \pm 0.009$ & 0.06 & & $\checkmark$ & & $\checkmark$ & $\checkmark$ & $\mathrm{C}$ \\
\hline I17220-3609 & 25.12 & 3.77 & - & 0.22 & $\checkmark$ & $\checkmark$ & & $\checkmark$ & $\checkmark$ & A \\
\hline I17233-3606 C1 & 39.81 & 4.91 & $8.93 \pm 0.712$ & 0.15 & $\checkmark$ & $\checkmark$ & $\checkmark$ & $\checkmark$ & $\checkmark$ & B \\
\hline $\mathrm{C} 2$ & 39.81 & 4.91 & $2.91 \pm 0.241$ & 0.01 & $\checkmark$ & $\checkmark$ & $\checkmark$ & $\checkmark$ & $\checkmark$ & A \\
\hline $\mathrm{C} 3$ & 39.81 & 4.91 & $1.87 \pm 0.235$ & 0.22 & $\checkmark$ & $\checkmark$ & & $\checkmark$ & $\checkmark$ & B \\
\hline I17244-3536 & 39.81 & 4.28 & $0.79 \pm 0.182$ & 0.16 & $\checkmark$ & & & & & B \\
\hline I17258-3637 C1 & 398.11 & 4.02 & - & 0.3 & $\checkmark$ & $\checkmark$ & & & $\checkmark$ & B \\
\hline $\mathrm{C} 2$ & 398.11 & 4.02 & - & 0.57 & $\checkmark$ & & & & & B \\
\hline I17269-3312 & 12.59 & 3.66 & $25.06 \pm 2.55$ & 0.04 & & $\checkmark$ & $\checkmark$ & $\checkmark$ & $\checkmark$ & $\mathrm{C}$ \\
\hline I17271-3439 & 39.81 & 4.23 & $1.16 \pm 0.094$ & 0.1 & $\checkmark$ & $\checkmark$ & & $\checkmark$ & $\checkmark$ & A \\
\hline $\mathrm{I} 17278-3541 \mathrm{C} 1$ & 19.95 & 4.64 & - & 0.16 & & $\checkmark$ & & $\checkmark$ & $\checkmark$ & $\mathrm{D}$ \\
\hline $\mathrm{C} 2$ & 19.95 & 4.64 & - & 0.15 & & $\checkmark$ & & $\checkmark$ & $\checkmark$ & $\mathrm{D}$ \\
\hline $\mathrm{C} 3$ & 19.95 & 4.64 & $1.32 \pm 0.118$ & 0.25 & & & & & & $\mathrm{D}$ \\
\hline $\mathrm{C} 4$ & 19.95 & 4.64 & - & 0.21 & & $\checkmark$ & & & $\checkmark$ & $\mathrm{D}$ \\
\hline C5 & 19.95 & 4.64 & - & 0.08 & & & $\checkmark$ & $\checkmark$ & $\checkmark$ & $\mathrm{D}$ \\
\hline C6 & 19.95 & 4.64 & - & 0.02 & & $\checkmark$ & $\checkmark$ & & $\checkmark$ & $\mathrm{C}$ \\
\hline I17439-2845 & 79.43 & 3.66 & - & 1.39 & $\checkmark$ & & & $\checkmark$ & $\checkmark$ & B \\
\hline I17441-2822 C1 & 15.85 & 4.81 & $0.6 \pm 0.063$ & 1.37 & $\checkmark$ & - & - & - & & B \\
\hline $\mathrm{C} 2$ & 15.85 & 4.81 & $0.52 \pm 0.046$ & 0.96 & $\checkmark$ & - & - & - & & B \\
\hline
\end{tabular}




\begin{tabular}{|c|c|c|c|c|c|c|c|c|c|c|}
\hline \multirow[t]{3}{*}{ IRAS } & \multirow{3}{*}{$\begin{array}{c}L_{\mathrm{bol}} / M \\
\left(L_{\odot} M_{\odot}^{-1}\right)\end{array}$} & \multirow{3}{*}{$\begin{array}{c}\log n \\
\left(\mathrm{~cm}^{-3}\right)\end{array}$} & \multirow[t]{3}{*}[\mathrm{SiO}]{$/\left[\mathrm{H}^{13} \mathrm{CO}^{+}\right]$} & \multirow[t]{2}{*}{$D_{\mathrm{d}}$} & \multirow[t]{3}{*}{$\mathrm{H} 40 \alpha$} & \multicolumn{3}{|c|}{ Wing } & \multirow[t]{3}{*}{ outflow } & \multirow[t]{3}{*}{ Type } \\
\hline & & & & & & $\mathrm{SiO}$ & $\mathrm{HCO}^{+}$ & $\mathrm{CS}$ & & \\
\hline & & & & $(\mathrm{pc})$ & & & & & & \\
\hline $\mathrm{C} 3$ & 15.85 & 4.81 & $0.28 \pm 0.057$ & 1.13 & $\checkmark$ & - & - & - & & B \\
\hline $\mathrm{C} 4$ & 15.85 & 4.81 & $0.16 \pm 0.017$ & 1.26 & $\checkmark$ & - & - & - & & B \\
\hline $\mathrm{C} 5$ & 15.85 & 4.81 & - & 0.36 & $\checkmark$ & - & - & - & & B \\
\hline I17455-2800 & 63.1 & 3.7 & $3 \pm 0.401$ & 0.78 & $\checkmark$ & $\checkmark$ & & & $\checkmark$ & B \\
\hline I17545-2357 & 10.0 & 3.89 & - & 0.49 & $\checkmark$ & & & & & B \\
\hline I17589-2312 C1 & 10.0 & 4.17 & - & 0.45 & & & & & & $\mathrm{D}$ \\
\hline $\mathrm{C} 2$ & 10.0 & 4.17 & $1.16 \pm 0.105$ & 0.23 & & & & & & $\mathrm{D}$ \\
\hline I18032-2032 & 79.43 & 3.8 & $2.45 \pm 0.016$ & 0.24 & $\checkmark$ & $\checkmark$ & $\checkmark$ & $\checkmark$ & $\checkmark$ & B \\
\hline I18056-1952 C1 & 19.95 & 3.92 & $1.02 \pm 0.017$ & 0 & $\checkmark$ & $\checkmark$ & $\checkmark$ & $\checkmark$ & $\checkmark$ & A \\
\hline $\mathrm{C} 2$ & 19.95 & 3.92 & $2.08 \pm 0.012$ & 1.55 & $\checkmark$ & $\checkmark$ & $\checkmark$ & $\checkmark$ & $\checkmark$ & B \\
\hline $\mathrm{C} 3$ & 19.95 & 3.92 & $0.39 \pm 0.026$ & 0.73 & $\checkmark$ & $\checkmark$ & & & $\checkmark$ & B \\
\hline I18079-1756 C1 & 19.95 & 4.28 & $0.34 \pm 0.026$ & 0.05 & & & & & & $\mathrm{C}$ \\
\hline $\mathrm{C} 2$ & 19.95 & 4.28 & $0.47 \pm 0.099$ & 0.26 & & & & & & $\mathrm{D}$ \\
\hline I18089-1732 & 15.85 & 3.85 & $0.62 \pm 0.069$ & 0.03 & & $\checkmark$ & $\checkmark$ & $\checkmark$ & $\checkmark$ & $\mathrm{C}$ \\
\hline I18110-1854 & 39.81 & 3.99 & $0.87 \pm 0.13$ & 0.35 & $\checkmark$ & $\checkmark$ & & & $\checkmark$ & B \\
\hline I18116-1646 & 100.0 & 3.73 & $0.09 \pm-$ & 0.18 & $\checkmark$ & & & & & B \\
\hline I18117-1753 & 12.59 & 4.09 & $1.52 \pm 0.171$ & 0.11 & & $\checkmark$ & $\checkmark$ & $\checkmark$ & $\checkmark$ & $\mathrm{D}$ \\
\hline I18139-1842 & 251.19 & 4.15 & $0.6 \pm 0.103$ & 0.14 & $\checkmark$ & $\checkmark$ & & & $\checkmark$ & B \\
\hline I18159-1648 & 10.0 & 4.37 & $2.31 \pm 0.259$ & 0.03 & & $\checkmark$ & $\checkmark$ & $\checkmark$ & $\checkmark$ & $\mathrm{C}$ \\
\hline I18182-1433 & 15.85 & 4.16 & $0.92 \pm 0.08$ & 0.04 & & $\checkmark$ & & & $\checkmark$ & $\mathrm{C}$ \\
\hline I18223-1243 & 19.95 & 3.76 & $2.47 \pm 0.68$ & 0.81 & & & $\checkmark$ & & $\checkmark$ & $\mathrm{D}$ \\
\hline I18228-1312 & 50.12 & 3.89 & $0.57 \pm 0.24$ & 0.19 & $\checkmark$ & & & & & B \\
\hline I18236-1205 & 3.98 & 4.14 & $0.71 \pm 0.153$ & 0.11 & & $\checkmark$ & & $\checkmark$ & $\checkmark$ & $\mathrm{D}$ \\
\hline I18264-1152 & 5.01 & 4.09 & $1.24 \pm 0.015$ & 0.14 & & $\checkmark$ & $\checkmark$ & $\checkmark$ & $\checkmark$ & $\mathrm{D}$ \\
\hline I18290-0924 & 6.31 & 3.7 & $1.59 \pm 0.245$ & 0.24 & & & & & & $\mathrm{D}$ \\
\hline I18311-0809 & 19.95 & 3.33 & $1.05 \pm 0.091$ & 0.12 & $\checkmark$ & $\checkmark$ & $\checkmark$ & $\checkmark$ & $\checkmark$ & A \\
\hline I18316-0602 C1 & 12.59 & 4.27 & $42.04 \pm 1.271$ & 0.09 & & $\checkmark$ & & $\checkmark$ & $\checkmark$ & $\mathrm{D}$ \\
\hline $\mathrm{C} 2$ & 12.59 & 4.27 & $1.53 \pm 0.103$ & 0.17 & & $\checkmark$ & $\checkmark$ & $\checkmark$ & $\checkmark$ & $\mathrm{D}$ \\
\hline $\mathrm{C} 3$ & 12.59 & 4.27 & - & 0.07 & & & & $\checkmark$ & $\checkmark$ & $\mathrm{C}$ \\
\hline I18341-0727 & 25.12 & 3.4 & $1.49 \pm 0.084$ & 0.45 & $\checkmark$ & $\checkmark$ & $\checkmark$ & $\checkmark$ & $\checkmark$ & B \\
\hline I18411-0338 & 25.12 & 3.42 & $0.42 \pm 0.002$ & 0.07 & & $\checkmark$ & & & $\checkmark$ & $\mathrm{C}$ \\
\hline I18434-0242 & 125.89 & 3.7 & $1.04 \pm 0.026$ & 0.2 & $\checkmark$ & $\checkmark$ & & $\checkmark$ & $\checkmark$ & A \\
\hline I18461-0113 & 19.95 & 4.27 & $0.73 \pm 0.004$ & 0.1 & & $\checkmark$ & $\checkmark$ & $\checkmark$ & $\checkmark$ & $\mathrm{C}$ \\
\hline I18469-0132 & 63.1 & 4.12 & $0.34 \pm 0.002$ & 0.44 & & $\checkmark$ & & & $\checkmark$ & $\mathrm{D}$ \\
\hline I18479-0005 & 79.43 & 3.65 & $0.56 \pm 0.027$ & 0.12 & $\checkmark$ & & & & & A \\
\hline I18507p0110 C1 & 39.81 & 4.88 & $0.75 \pm 0.008$ & 0.03 & $\checkmark$ & $\checkmark$ & & $\checkmark$ & $\checkmark$ & A \\
\hline $\mathrm{C} 2$ & 39.81 & 4.88 & - & 0.24 & $\checkmark$ & $\checkmark$ & $\checkmark$ & $\checkmark$ & $\checkmark$ & B \\
\hline $\mathrm{C} 3$ & 39.81 & 4.88 & - & 0.18 & $\checkmark$ & & & & & $\mathrm{B}$ \\
\hline I18507p0121 C1 & 7.94 & 4.83 & $3.42 \pm 0.067$ & 0.01 & & $\checkmark$ & $\checkmark$ & $\checkmark$ & $\checkmark$ & $\mathrm{C}$ \\
\hline $\mathrm{C} 2$ & 7.94 & 4.83 & $3.42 \pm 0.399$ & 0.12 & & $\checkmark$ & & $\checkmark$ & $\checkmark$ & $\mathrm{D}$ \\
\hline I18517p0437 & 50.12 & 5.43 & $0.5 \pm 0.022$ & 0.05 & & & & & & $\mathrm{C}$ \\
\hline $\mathrm{I} 18530 \mathrm{p} 0215$ & 25.12 & 3.84 & $0.13 \pm 0.029$ & 0.23 & $\checkmark$ & $\checkmark$ & & & $\checkmark$ & $\mathrm{B}$ \\
\hline I19078p0901 & 79.43 & 3.73 & $2.12 \pm 0.02$ & 0.16 & $\checkmark$ & $\checkmark$ & $\checkmark$ & $\checkmark$ & $\checkmark$ & A \\
\hline I19095p0930 & 100.0 & 4.29 & $0.8 \pm 0.014$ & 0.06 & & $\checkmark$ & $\checkmark$ & $\checkmark$ & $\checkmark$ & $\mathrm{C}$ \\
\hline I19097p0847 & 15.85 & 3.5 & $1.18 \pm 0.166$ & 0.31 & & $\checkmark$ & $\checkmark$ & $\checkmark$ & $\checkmark$ & $\mathrm{D}$ \\
\hline
\end{tabular}

Note. $L_{\mathrm{bol}} / M$ : The bolometric luminosity to mass ratio. $n$ : The particle number density. $[\mathrm{SiO}] /\left[\mathrm{H}^{13} \mathrm{CO}^{+}\right]$: The integrated intensity ratio of $\mathrm{SiO}$ emission and $\mathrm{H}^{13} \mathrm{CO}^{+}$emssion, and the - symbol indicates that the source has absorption features in their spectra. $D_{\mathrm{d}}$ : The separated linear distance between the central position of $\mathrm{SiO}$ clumps and the peak position of the $3 \mathrm{~mm}$ continuum emission. $\mathrm{H} 40 \alpha$ column: the $\checkmark$ symbol indicates that the $\mathrm{SiO}$ clump is associated with $\mathrm{H} 40 \alpha$ emission. Wing columns: The $\checkmark$ symbol represents that the SiO clump shows line wing emission; the sources with - symbol are excluded in analysis. outflow column: The $\checkmark$ symbol indicates that the $\mathrm{SiO}$ clump is with outflow. Type column: A is the $\mathrm{SiO}$ clumps associated with both $\mathrm{H} 40 \alpha$ emission and $3 \mathrm{~mm}$ continuum emission. B is the $\mathrm{SiO}$ clumps separated from both $\mathrm{H} 40 \alpha$ emission and $3 \mathrm{~mm}$ continuum emission. $\mathrm{C}$ is the $\mathrm{SiO}$ clumps associated with $3 \mathrm{~mm}$ continuum emission undetected $\mathrm{H} 40 \alpha$ emission. D is the $\mathrm{SiO}$ clumps partly separated from $3 \mathrm{~mm}$ continuum emission undetected $\mathrm{H} 40 \alpha$ emission. 